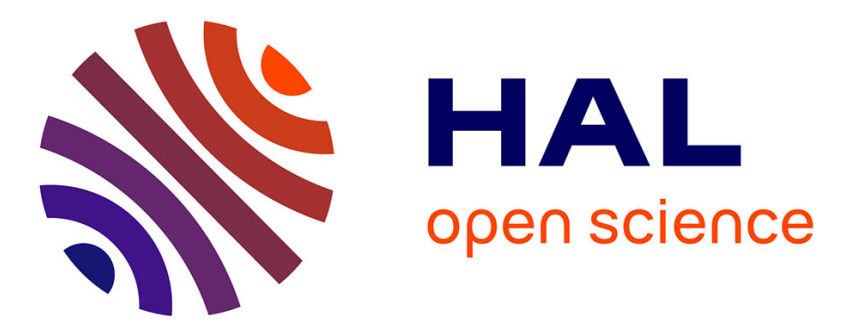

\title{
Analysis of PWA control of discrete-time linear dynamics in the presence of variable input delay
}

Mohammed-Tahar Laraba, Sorin Olaru, Silviu-Iulian Niculescu

\section{To cite this version:}

Mohammed-Tahar Laraba, Sorin Olaru, Silviu-Iulian Niculescu. Analysis of PWA control of discretetime linear dynamics in the presence of variable input delay. IFAC Journal of Systems and Control, 2017, 1, pp.24 - 36. 10.1016/j.ifacsc.2017.06.002 . hal-01719825

\section{HAL Id: hal-01719825 \\ https://hal-centralesupelec.archives-ouvertes.fr/hal-01719825}

Submitted on 2 Mar 2018

HAL is a multi-disciplinary open access archive for the deposit and dissemination of scientific research documents, whether they are published or not. The documents may come from teaching and research institutions in France or abroad, or from public or private research centers.
L'archive ouverte pluridisciplinaire HAL, est destinée au dépôt et à la diffusion de documents scientifiques de niveau recherche, publiés ou non, émanant des établissements d'enseignement et de recherche français ou étrangers, des laboratoires publics ou privés. 


\title{
Analysis of PWA control of discrete-time linear dynamics in the presence of variable input delay
}

\author{
Mohammed-Tahar Laraba, Sorin Olaru, and Silviu-Iulian Niculescu
}

\begin{abstract}
This paper focuses on the robustness of a specific class of control laws, namely the piecewise affine (PWA) controllers, defined over a bounded region of the state-space. More precisely, we are interested in closed-loop systems emerging from linear dynamical systems controlled via feedback channels in the presence of varying transmission delays by a PWA controller defined over a polyhedral partition of the state-space. We exploit the fact that the variable delays are inducing some particular model uncertainty. Our objective is to characterize the delay invariance margins: the collection of all possible values of the time-varying delays for which the positive invariance of the corresponding region is guaranteed with respect to the closedloop dynamics. These developments are proving to be useful for the analysis of different design methodologies and, in particular, for model predictive control (MPC) approaches. The proposed delay margin describes the admissible transmission delays for an MPC implementation. From a different perspective, the delay margin further characterizes the fragility of an embedded MPC implementation via the on-line optimization and subject to variable computational time.
\end{abstract}

Index Terms-Time-delay systems, Model Predictive Control, PWA controller, Delay margins.

\section{INTRODUCTION}

$\mathbf{T}$ IME-delay appears naturally in modeling dynamics or networked control systems (NCSs) as well as many other physical processes where propagation and transport phenomena occur. It is worth mentioning that the presence of communication networks in the closed-loop control systems induces varying transmission delays [1]. These delays are known to degrade the control performance and can induce instability as documented in the rich theoretic control literature dedicated to such topics [2], [3].

Model Predictive Control (MPC) represents a well-known control technique which, roughly speaking, solves an on-line constrained optimization problem over a receding horizon [4], [5]. It constructs at each sampling instant an optimal sequence with respect to an appropriate performance index. Unfortunately, using MPC in the presence of time-varying delays leads to complex optimization problems, which are difficult to handle from the numerical point of view. Linear MPC with constraints is known to result in PWA closed-loop dynamics [6], [7].

Checking the stability of the fixed points for a PWA system is not an easy task in general even in the absence of transmission delay. Stability of PWA systems has been investigated in the literature using Piecewise Quadratic Lyapunov functions

\footnotetext{
All authors are with the Laboratory of Signals and Systems of CentraleSupélec, Paris-Saclay University, 91192 Gif-SurYvette, France: mohammed.laraba, sorin.olaru, silviu.niculescullss.supelec.fr.
}

[8]. The stability test is usually formulated in terms of linear matrix inequalities (LMIs), that obviously lead to sufficient conditions. Due to the conservatism of such approaches, alternative relaxations can be found in [9], [10].

To the best of the authors' knowledge, when dealing with positive invariance of sets [11], [12] with respect to delay difference equations (DDE), two main approaches exist. The first one relies on the fact that the DDE allows a finite dimensional extended state space model construction. This extended state space leads to an invariant set characterization with respect to an equivalent linear time invariant model. The second approach aims to obtain an invariant set with respect to a dynamical system in the original state space of DDE, which is independent from the delay value. This concept is called $\mathcal{D}$-invariance [13].

In this paper, we will conduct a robustness analysis of discrete-time linear dynamics in closed-loop with a PWA control law in the presence of time-varying input delays affecting the communication on the feedback channel or induced by the control computation itself. The PWA feedback is generic but it can be obtained, for example, by using an explicit MPC design constructed upon the nominal delay-free model. We show that invariance analysis is fundamental when a PWA control law is constructed without a-priori taking the delay into account. The presence of variable input delay induces a parametric uncertainty in the closed-loop parameters. One way to handle this parametric uncertainty is to cover all possible delay variations by embedding it within a polytopic model when the maximal delay is known [1], [14]-[16].

The contribution of the paper is threefold: first, a formal definition of delay margins based on positive invariance is given. Its structure and computations are detailed providing an important tool for the analysis of this class of closed-loop systems. Second, the inverse problem of finding the maximal range of delay variation, guaranteeing the invariance of the region over which the PWA control law structure is specified, represents, to the best of the authors' knowledge, an open problem and will receive in the sequel a complete characterization. Third, we are proposing a constructive method to find the delay margins based on a positive invariance approach. It is important to point out that the notion of delay margin is introduced in terms of sets and mappings generalizing the underlying idea of the first delay interval and its related delay margins from continuous-time systems. We first investigate the case when the PWA control input is subject to an intersample delay variation, i.e. the delay variation bounds remain inside a sampling period. The procedure describes, by means of set projections, all possible delay values for which the positive invariance (or alternatively $\mathcal{D}$-invariance) of the state trajecto- 
ries is guaranteed. The relationship between delay margins in different state space representations is established and linked to set factorization [17]. Furthermore, we extend the delay margins procedure to the multisample case i.e. the delay variation bounds are larger than one sampling period. The paper makes use of some preliminary results in [18], [19] and extends the robustness analysis to PWA systems with variable input delays.

The paper is structured as follows: section II presents some preliminary mathematical notions and definitions related to piecewise affine functions and positive invariance for discretetime systems. Two classes of linear continuous time-invariant systems affected by variable delay are introduced in section III. In the same section, the exact discretization of the considered dynamics and the uncertain PWA systems obtained in closedloop are discussed. Section IV is devoted to the construction of delay margins in order to ensure invariance in the presence of small and large delays. Finally, an illustrative example is shown in section $\mathrm{V}$ and concluding remarks are drawn in section VI.

Notations: We denote by $\mathbb{R}\left(\mathbb{R}_{+}\right), \mathbb{N}$ and $\mathbb{Z}\left(\mathbb{Z}_{+}\right)$the field of real (non-negative real) numbers, the set of non-negative integers, the set of integer (strictly positive integer) numbers, respectively. For every interval $\Pi$ of $\mathbb{R}$ we define $\mathbb{Z}_{\Pi}:=\mathbb{Z} \cap \Pi$. Given $m \in \mathbb{Z}_{+}$, by $\mathbb{1}_{m}$, we denote the vector of dimension $m$ with all the entries equal to 1 and, by $I_{m} \in \mathbb{R}^{m \times m}$, the $m \times m$ identity matrix. Conv denotes the convex hull operation, and $\otimes$ the Kronecker product of two matrices. In the sequel and in order to avoid ambiguity, whenever an exponent is associated with a matrix, it will be interpreted as a matrix raised to a power or just as an index depending on the context.

\section{PRELIMINARIES AND PREREQUISITES}

In this section we discuss in a brief manner, some basic concepts related to piecewise affine functions and positive invariance for discrete time-varying systems.

Given two sets $\mathcal{X}, \mathcal{Y} \subset \mathbb{R}^{m}, \mathcal{X} \oplus \mathcal{Y}$ and $\mathcal{X} \times \mathcal{Y}$ denote the Minkowski sum and the Cartesian product of these two sets, respectively, defined as follows:

$$
\begin{gathered}
\mathcal{X} \oplus \mathcal{Y}:=\{z \mid \exists(x, y) \in(\mathcal{X}, \mathcal{Y}) \text { such that } z=x+y\} . \\
\mathcal{X} \times \mathcal{Y}:=\{(x, y) \mid x \in \mathcal{X} \text { and } y \in \mathcal{Y}\} .
\end{gathered}
$$

The unit simplex in $\mathbb{R}^{m}$ is defined as:

$$
S_{m}:=\left\{x \in \mathbb{R}_{+}^{m} \mid \mathbb{1}_{m}^{T} x=1\right\} .
$$

Given a map $f: \mathbb{R}^{n} \rightarrow \mathbb{R}^{m}$ and a set $\mathcal{S} \subset \mathbb{R}^{n}$, we denote the image of the set $\mathcal{S}$ by the mapping $f(\cdot)$ :

$$
f(\mathcal{S}):=\left\{y \in \mathbb{R}^{m} \mid \exists x \in \mathcal{S} \text { such that } y=f(x)\right\}
$$

In the particular case of affine mappings $f(x)=A x+B, A \in$ $\mathbb{R}^{m \times n}, B \in \mathbb{R}^{m}$, the image of a set $\mathcal{S} \subset \mathbb{R}^{n}$ is written $f(\mathcal{S})=$ $A \mathcal{S}+B$. For a given set $\mathcal{X} \subseteq \mathcal{Y} \times \mathcal{Z}$, int $(\mathcal{X})$ denotes the interior of $\mathcal{X}$, the projection of $\mathcal{X}$ onto $\mathcal{Y}$ is defined as:

$$
\operatorname{Proj}_{\mathcal{Y}} \mathcal{X}=\{y \in \mathcal{Y} \mid \exists z \in \mathcal{Z} \text { such that }(y, z) \in \mathcal{X}\} \text {. }
$$

The notions of state-space partition, PWA functions and positive invariance are the classical ones as defined, for example, in [18], [20].

Definition 2.1: Let $\mathcal{X}$ be a compact subset of $\mathbb{R}^{n}$. A partition of $\mathcal{X}$ is a finite family of subsets $\mathcal{X}_{i} \subset \mathbb{R}^{n}$ of $\mathcal{X}$, with $i \in \mathcal{I}_{N} \subset \mathbb{Z}_{+}$being the index of the regions and $\mathcal{I}_{N}$ a finite subset of $\mathbb{Z}_{+}$, which verifies the following conditions:

- $\operatorname{int}\left(\mathcal{X}_{i}\right) \neq \emptyset$ for $i \in \mathcal{I}_{N} \subset \mathbb{Z}_{+}$.

- The regions $\mathcal{X}_{i}$ are said to cover $\mathcal{X}$, i.e.

$$
\mathcal{X}=\bigcup_{i \in \mathcal{I}_{N}} \mathcal{X}_{i}
$$

- The elements of $\mathcal{X}_{i}$ are pairwise disjoint i.e.

$$
\operatorname{int}\left(\mathcal{X}_{i} \cap \mathcal{X}_{j}\right)=\emptyset \text { for }(i, j) \in \mathcal{I}_{N}^{2} \text { and } i \neq j .
$$

Two elements $\mathcal{X}_{i}$ and $\mathcal{X}_{j},(i, j) \in \mathcal{I}_{N}^{2}, i \neq j$, of the partition for which $\operatorname{dim}\left(\mathcal{X}_{i} \cap \mathcal{X}_{j}\right)=n-1$ are called neighbors.

Definition 2.2: A function $f^{p w a}: \mathcal{X} \rightarrow \mathbb{R}^{m}$ defined over a polyhedral partition $\mathcal{X}=\bigcup_{i \in \mathcal{I}_{N}} \mathcal{X}_{i}$ by the relation:

$$
f^{p w a}(x)=A_{i} x+a_{i} \text { for } i \text { such that } x \in \mathcal{X}_{i}
$$

where $A_{i} \in \mathbb{R}^{m \times n}$ and $a_{i} \in \mathbb{R}^{m}$ is called piecewise affine function over $\mathcal{X} \subset \mathbb{R}^{n}$.

Definition 2.3: A piecewise affine function $f^{p w a}(\cdot)$ defined over a polyhedral partition of a polyhedron $\mathcal{X}$ is continuous if and only if the equality:

$$
A_{i} x+a_{i}=A_{j} x+a_{j}
$$

holds $\forall x \in \mathcal{X}_{i} \cap \mathcal{X}_{j},(i, j) \in \mathcal{I}_{N}^{2}, i \neq j$ and $\operatorname{dim}\left(\mathcal{X}_{i} \cap \mathcal{X}_{j}\right)=$ $n-1$.

Definition 2.4: A set $\mathcal{X} \subset \mathbb{R}^{n}$ is positively invariant with respect to the dynamical system $x_{k+1}=f\left(k, x_{k}\right)$ if for any initial condition $x_{0} \in \mathcal{X}$ the state trajectory satisfies $x_{k} \in$ $\mathcal{X}, \forall k \in \mathbb{Z}_{+}$.

Definition 2.5: A set $\mathcal{X} \subset \mathbb{R}^{n}$ is called $\mathcal{D}$-invariant with respect to the linear dynamics:

$$
x_{k+1}=\sum_{i=0}^{d} A_{i} x_{k-i}
$$

with initial conditions $x_{-i} \in \mathcal{X}$ for all $i \in \mathbb{Z}_{[0, d]}$ if the state trajectory satisfies $x_{k} \in \mathcal{X}, \forall k \in \mathbb{Z}_{+}$. This is equivalent using the Minkowski addition to $\bigoplus_{i=0}^{d} A_{i} \mathcal{X} \subseteq \mathcal{X}$.

Several properties govern the relationship between $\mathcal{D}$-invariant sets. One of these properties, known as the delay independent property, is presented in the following proposition:

Proposition 2.6: [13] If the set $\mathcal{X} \subset \mathbb{R}^{n}$ is $\mathcal{D}$-invariant with respect to (3) then $\mathcal{X}$ is $\mathcal{D}$-invariant for:

$$
x_{k+1}=\sum_{i=0}^{d} A_{i} x_{k-\tau_{i}}
$$

for any $\tau_{i} \in \mathbb{Z}_{+}$.

This property, proven in [13], will be used later in the development of the main result related to delay margins for large delays. 


\section{DYNAMICAL MODEL OF A LINEAR PLANT WITH DIGITAL CONTROL IN THE PRESENCE OF VARIABLE INPUT-DELAY}

\section{A. Intersample delays}

For the sake of simplicity of the presentation, we discuss first the case when the delay variation is intersample, i.e. smaller than or equal to the sampling period.

1) System dynamics: Consider a linear continuous timeinvariant (LTI) system and a sequence of delays $\left(\tau_{k}\right)$ affecting the input as follows:

$$
\left\{\begin{array}{l}
\dot{x}(t)=A_{c} x(t)+B_{c} u(t) \\
u(t)=u_{k}, \forall t \in\left[t_{k}+\tau_{k}, t_{k+1}+\tau_{k+1}\right) .
\end{array}\right.
$$

where $A_{c} \in \mathbb{R}^{n \times n}, B_{c} \in \mathbb{R}^{n \times m}, x(t) \in \mathbb{R}^{n}$ the continuous system state. Moreover, assume that the system states are sampled periodically with the period $T_{s} \in \mathbb{R}_{+}^{*}$ and we denote by $t_{k}=k T_{s}$ the $k^{t h}$ sampling instant. The control input $u(t) \in \mathbb{R}^{m}$ is known for $t \in\left[0, \tau_{0}\right)$, and the control action generated at time $t=t_{k}$ at the controller side is denoted by $u_{k} \in \mathbb{R}^{m}$. The possible delay induced by the network at sample instant $t_{k}$ is denoted by $\tau_{k} \in[\underline{\tau}, \bar{\tau}]$, with a lower bound $\underline{\tau} \in \mathbb{R}_{[0, \bar{\tau}]}$ and an upper bound $\bar{\tau} \in \mathbb{R}_{\left[\underline{\tau}, T_{s}\right]}$. We will recall next the modeling in discrete-time following the approach in the studies [1], [14], [16]. We consider the exact discretization of (5) by exploiting the fact that the control action is piecewise constant, i.e. $u(t)=u_{k}, \forall t \in\left[t_{k}+\tau_{k}, t_{k+1}+\tau_{k+1}\right)$ :

$$
\begin{array}{r}
x_{k+1}=e^{A_{c} T_{s}} x_{k}+\int_{0}^{\tau_{k}} e^{A_{c}\left(T_{s}-\theta\right)} d \theta B_{c} u_{k-1}+ \\
\int_{\tau_{k}}^{T_{s}} e^{A_{c}\left(T_{s}-\theta\right)} d \theta B_{c} u_{k}
\end{array}
$$

and let $\epsilon_{k}=T_{s}-\tau_{k}$, and:

$$
\begin{gathered}
A=e^{A_{c} T_{s}}, B=\int_{0}^{T_{s}} e^{A_{c}\left(T_{s}-\theta\right)} d \theta B_{c} \\
\Delta\left(\epsilon_{k}\right)=\int_{T_{s}-\epsilon_{k}}^{T_{s}} e^{A_{c}\left(T_{s}-\theta\right)} d \theta B_{c}=\int_{-\epsilon_{k}}^{0} e^{-A_{c} \sigma} d \sigma B_{c} .
\end{gathered}
$$

Then, the discrete-time model which takes into account the effect of the continuous time-delay variation will become:

$$
x_{k+1}=A x_{k}+\left(B-\Delta\left(\epsilon_{k}\right)\right) u_{k-1}+\Delta\left(\epsilon_{k}\right) u_{k} .
$$

In the general case, the variable time-delay implies a variable limit ' $\epsilon_{k}$ ' for the integration in (8). One can see that there is no explicit link between the samples available for the discrete model and the delay in continuous-time, thus leading practically to a parameter-varying dynamical model. Upon discretization, the variable input delay is considered in terms of an appropriate uncertainty function. All possible delay variations can be covered by confining the induced model uncertainty within a polytopic description. Therefore, a polytopic (simplicial) over-approximation of the uncertainty coming from the variable delay can be constructed (see, e.g. [1], [15], [16], [21]) to obtain finally an appropriate polytopic model. In a probabilistic framework, the delay variation can be considered uniformly distributed between the extreme realizations of the discrete-time model (in the present work, no other specific statistical information with respect to the delay variation is considered to be available).

It is interesting to note that by setting $\epsilon_{k}=0$ and $\epsilon_{k}=T_{s}$, we obtain two "extreme" realizations of the discrete-time model (9):

$$
x_{k+1}=A x_{k}+B u_{k-1}
$$

and

$$
\begin{gathered}
x_{k+1}=A x_{k}+\left(B-\Delta\left(T_{s}\right)\right) u_{k-1}+\Delta\left(T_{s}\right) u_{k} \\
=A x_{k}+B u_{k}
\end{gathered}
$$

respectively.

2) The PWA closed-loop dynamics: The starting point for the present work will be the nominal dynamics corresponding to $\epsilon_{k}=T_{s}$ (no delay induced by the network). A piecewise affine control law is designed with respect to this nominal dynamics. In this context, the following piecewise affine (PWA) control law can be obtained from an explicit constrained MPC design [22] for instance:

$$
\begin{aligned}
& u^{p w a}: \mathcal{X} \longrightarrow \mathbb{R}^{m} \\
& u^{p w a}(x)=F_{i} x+g_{i}, \forall i \in \mathcal{I}_{N} \text { s.t } x \in \mathcal{X}_{i} .
\end{aligned}
$$

We introduce the following:

Assumption 3.1: The set $\mathcal{X}$ is positive invariant with respect to the closed-loop nominal dynamics $x_{k+1}=A x_{k}+B u_{k}^{p w a}$. The PWA control law obtained will be in turn used in practice for the control of the linear parameter-varying dynamics subject to variable delay (9). The closed-loop dynamics resulting from applying:

$$
\left\{\begin{array}{l}
u_{k}^{p w a}=F_{i} x_{k}+g_{i}, \text { for } x_{k} \in \mathcal{X}_{i} \\
u_{k-1}^{p w a}=F_{j} x_{k-1}+g_{j}, \text { for } x_{k-1} \in \mathcal{X}_{j}
\end{array}\right.
$$

will be:

$$
\begin{gathered}
x_{k+1}=A x_{k}+\left(B-\Delta\left(\epsilon_{k}\right)\right)\left[F_{j} x_{k-1}+g_{j}\right]+ \\
\Delta\left(\epsilon_{k}\right)\left[F_{i} x_{k}+g_{i}\right] \\
=\left(A+\Delta\left(\epsilon_{k}\right) F_{i}\right) x_{k}+\left(B-\Delta\left(\epsilon_{k}\right)\right) F_{j} x_{k-1}+ \\
B g_{j}+\Delta(\epsilon)\left(g_{i}-g_{j}\right), \\
\forall(i, j) \in \mathcal{I}_{N}^{2} \text { such that } x_{k} \in \mathcal{X}_{i}, x_{k-1} \in \mathcal{X}_{j} .
\end{gathered}
$$

It is clear that an extended state-space representation can be constructed for the delay difference equation (14) by introducing an augmented state vector, i.e. $\xi_{k}=\left[x_{k}^{T} x_{k-1}^{T}\right]^{T} \in \mathbb{R}^{2 n}$. An equivalent state-space model is then obtained:

$$
\begin{array}{r}
\underbrace{\left[\begin{array}{c}
x_{k+1} \\
x_{k}
\end{array}\right]}_{\xi_{k+1}}=\left[\begin{array}{cc}
A+\Delta\left(\epsilon_{k}\right) F_{i} & \left(B-\Delta\left(\epsilon_{k}\right)\right) F_{j} \\
I_{n} & 0_{n \times n}
\end{array}\right] \underbrace{\left[\begin{array}{c}
x_{k} \\
x_{k-1}
\end{array}\right]}_{\xi_{k}}+ \\
\quad\left[\begin{array}{c}
B g_{j}+\Delta\left(\epsilon_{k}\right)\left(g_{i}-g_{j}\right) \\
0_{n \times 1}
\end{array}\right] ; \\
\forall(i, j) \in \mathcal{I}_{N}^{2} \text { such that } x_{k} \in \mathcal{X}_{i} \text { and } x_{k-1} \in \mathcal{X}_{j} .
\end{array}
$$


The difference equations (14) and (15) depend on $\Delta\left(\epsilon_{k}\right)$, considered as a parameter-varying matrix, lying in a nonconvex subset of $\mathbb{R}^{n \times m}$. One can write equations (14) and (15) in a more compact form as follows:

$$
\begin{array}{r}
x_{k+1}=\phi_{i}\left(\epsilon_{k}\right) x_{k}+\theta_{j}\left(\epsilon_{k}\right) x_{k-1}+\gamma_{i j}\left(\epsilon_{k}\right), \\
\forall(i, j) \in \mathcal{I}_{N}^{2} \text { s.t } x_{k} \in \mathcal{X}_{i} \text { and } x_{k-1} \in \mathcal{X}_{j} .
\end{array}
$$

where:

$$
\begin{aligned}
& \phi_{i}\left(\epsilon_{k}\right)=\left(A+\Delta\left(\epsilon_{k}\right) F_{i}\right) \\
& \theta_{j}\left(\epsilon_{k}\right)=\left(B-\Delta\left(\epsilon_{k}\right)\right) F_{j} \\
& \gamma_{i j}\left(\epsilon_{k}\right)=B g_{j}+\Delta\left(\epsilon_{k}\right)\left(g_{i}-g_{j}\right),
\end{aligned}
$$

or alternatively for (15) as:

$$
\left\{\begin{array}{l}
\xi_{k+1}=\Phi_{i j}\left(\epsilon_{k}\right) \xi_{k}+\Gamma_{i j}\left(\epsilon_{k}\right) \\
\forall(i, j) \in \mathcal{I}_{N}^{2} \text { such that } \xi_{k} \in \mathcal{X}_{i} \times \mathcal{X}_{j}
\end{array}\right.
$$

where:

$$
\begin{aligned}
\Phi_{i j}\left(\epsilon_{k}\right) & =\left[\begin{array}{cc}
A+\Delta\left(\epsilon_{k}\right) F_{i} & \left(B-\Delta\left(\epsilon_{k}\right)\right) F_{j} \\
I_{n} & 0_{n \times n}
\end{array}\right] \\
\Gamma_{i j}\left(\epsilon_{k}\right) & =\left[\begin{array}{c}
B g_{j}+\Delta\left(\epsilon_{k}\right)\left(g_{i}-g_{j}\right) \\
0_{n \times 1}
\end{array}\right]
\end{aligned}
$$

Moreover, one can define the following parameter-varying PWA mappings:

$$
\begin{aligned}
& \psi^{p w a}: \mathcal{X} \times \mathcal{X} \times \mathbb{R}_{\left[0, T_{s}\right]} \longrightarrow \mathbb{R}^{n} \\
& \left\{\begin{array}{l}
\psi^{p w a}(x, y, \epsilon)=\phi_{i}(\epsilon) x+\theta_{j}(\epsilon) y+\gamma_{i j}(\epsilon) \\
\forall(i, j) \in \mathcal{I}_{N}^{2} \text { such that } x \in \mathcal{X}_{i} \text { and } y \in \mathcal{X}_{j} .
\end{array}\right. \\
& \left\{\begin{array}{l}
\Psi^{p w a}(\xi, \epsilon)=\Phi_{i j}(\epsilon) \xi+\Gamma_{i j}(\epsilon) \\
\forall(i, j) \in \mathcal{I}_{N}^{2} \text { such that } \xi \in \mathcal{X}_{i} \times \mathcal{X}_{j} .
\end{array}\right.
\end{aligned}
$$

It is worth mentioning that the uncertain piecewise affine systems (16) and (18) are defined over the polyhedral partition of the compact sets $\mathcal{X}$ and $\mathcal{X} \times \mathcal{X}$ respectively, where the partition $\mathcal{X}=\bigcup_{i \in \mathcal{I}_{N}} \mathcal{X}_{i}$ is inherited from the explicit PWA control law design (12).

Now, the concept of delay margins with respect to dynamical systems, presented in the extended and the original statespace framework, is introduced in a set-theoretic perspective as follows:

Definition 3.2: The delay margin with respect to the timevarying dynamical system (16), denoted $d_{m}$, is given by:

$$
d_{\text {int }}=\left\{\epsilon \in\left[0, T_{s}\right] \mid \forall x \in \mathcal{X}_{i}, y \in \mathcal{X}_{j}, \psi^{p w a}(x, y, \epsilon) \in \mathcal{X}\right\} .
$$

Definition 3.3: The delay margin with respect to the timevarying dynamical system (18), denoted $\mathcal{D}_{m}$, is given by:

$$
\mathcal{D}_{\text {int }}=\left\{\epsilon \in\left[0, T_{s}\right] \mid \forall \xi \in \mathcal{X} \times \mathcal{X}, \Psi^{p w a}(\xi, \epsilon) \in \mathcal{X} \times \mathcal{X}\right\} .
$$

In continuous-time systems, the delay margin is classically denoting a scalar quantity representing the maximal delay preserving a certain dynamical property (asymptotic stability, marginal stability, etc). It allows defining an appropriate interval guaranteeing the corresponding property. Inspired by this last observation we choose to deal, in the present framework, with the delay in terms of admissible intervals (sets) of variations.

\section{B. Multisample delays: large delays and packet dropouts}

In this subsection, we extend the robustness problem formulation for a different class of dynamical systems, that is to say, discrete-time linear dynamics in closed-loop with a PWA control law defined over a polyhedral partition of the state space $\mathcal{X}$, in the presence of multisample delay variations. Multisample delay models are well suited when describing dynamics related to communication flows or propagation phenomena where the delay variation bounds are larger than one sampling period. In this case, discrete-time models that take into account the effect of the delay variation can be considered as a switched linear system as shown in the following.

1) System dynamics: Let us consider the linear continuous time-invariant system in the presence of time-varying input delay:

$$
\left\{\begin{array}{l}
\dot{x}(t)=A_{c} x(t)+B_{c} u(t) \\
u(t)=u_{k}, \forall t \in\left[t_{k}+\tau_{k}^{*}, t_{k+1}+\tau_{k+1}^{*}\right),
\end{array}\right.
$$

where $A_{c} \in \mathbb{R}^{n \times n}, B_{c} \in \mathbb{R}^{n \times m}, x(t) \in \mathbb{R}^{n}$ is the continuous system state vector. Since the controllers are implemented on digital platforms in many control applications, we assume that the plant is periodically sampled and actuated by the controller. The $k^{t h}$ sampling instants are denoted by $t_{k}=k T_{s}$. We denote by $\tau_{k}^{*} \in\left[\underline{\tau}^{*}, \bar{\tau}^{*}\right]$ the delay induced by the feedback communication channels and/or the computation time necessary for real-time control implementation. Moreover, we assume that the delay induced at sample instants $t_{k}$ has a lower bound $\underline{\tau}^{*} \in \mathbb{R}_{\left[0, \bar{\tau}^{*}\right]}$ and an upper bound $\bar{\tau}^{*} \in \mathbb{R}_{\left[\tau^{*}, T_{d}\right]}$ which can be larger than the sampling period $T_{d}>T_{s}$. The control input $u(t) \in \mathbb{R}^{m}$ is known for $t \in\left[0, \tau_{0}^{*}\right)$, and the control action generated at time $t=t_{k}$ at the controller side is denoted by $u_{k} \in \mathbb{R}^{m}$. One has to take into consideration the discrete-time delay variation induced by the network. We consider first the case $d_{\max }=d_{\min }+1$ and denote $d_{\max }=d$ for simplicity.

$$
(d-1) T_{s}<\tau_{k}^{*}<d T_{s}
$$

Next, consider the exact discretization of (22) assuming the control action is piecewise constant, i.e. $u(t)=u_{k}, \forall t \in$ $\left[t_{k}+\tau_{k}^{*}, t_{k+1}+\tau_{k+1}^{*}\right), \tau_{k}^{*}=(d-1) T_{s}+\tau_{k}$

$$
\begin{aligned}
x_{k+1}=e^{A_{c} T_{s}} x_{k}+ & \int_{0}^{\tau_{k}} e^{A_{c}\left(T_{s}-\theta\right)} d \theta B_{c} u_{k-d}+ \\
& \int_{\tau_{k}}^{T_{s}} e^{A_{c}\left(T_{s}-\theta\right)} d \theta B_{c} u_{k-d+1}
\end{aligned}
$$

and let $\epsilon_{k}=d T_{s}-\tau_{k}^{*}=T_{s}-\tau_{k}$, and based on the matrices introduced in (7)-(8), the discrete-time model which describes the presence of continuous time-delays variation, possibly larger than the sampling period $T_{s}$, will become:

$$
x_{k+1}=A x_{k}+B u_{k-d}-\Delta\left(\epsilon_{k}\right)\left(u_{k-d}-u_{k-d+1}\right)
$$

This model takes into account the effect of the delays which can be either smaller or larger than one sampling period. However, network data transmission channels can be unreliable, packet dropouts in the communication are inevitable. Control inputs can be lost during transmission and the structure of the discrete-time model has to consider both the networkinduced delays and data dropouts. The model (24) can be 
generalized by taking into account the previous aspects. Finally, the discrete-time model which considers the effect of the continuous-time delay variation larger than one sampling period and packets loss will be as follows:

$$
\left\{\begin{array}{l}
x_{k+1}=A x_{k}+B u_{k-d_{1}}-\Delta\left(\epsilon_{k}\right)\left(u_{k-d_{1}}-u_{k-d_{2}}\right) \\
\forall d_{1}, d_{2} \in\left\{d_{\min }, \cdots, d_{\max }\right\}, \text { and } d_{1}>d_{2}
\end{array}\right.
$$

The intersample delay variation induces some model uncertainties represented by the matrix $\Delta\left(\epsilon_{k}\right) \in \boldsymbol{\Delta}, k \in \mathbb{N}$ :

$$
\boldsymbol{\Delta}=\left\{\Delta\left(\epsilon_{k}\right) \mid \epsilon_{k} \in\left[0, T_{s}\right]\right\}
$$

Analytical methods exist when dealing with such model uncertainties as mentioned before. The key idea is to use the convex bounding of the matrix $\Delta\left(\epsilon_{k}\right)$ to embed it within a polytopic set of matrices with ' $s+1^{\prime}$ extreme realizations as follows:

$$
\forall \epsilon_{k} \in \mathbb{R}_{\left[0, T_{s}\right]}, \exists \alpha \in S_{s+1} \text { such that } \boldsymbol{\Delta}=\sum_{i=0}^{s} \alpha_{i} \Delta_{i} .
$$

2) The PWA closed-loop dynamics: The starting point of the analysis in this case is the following uncertain delay difference equation:

$$
\left\{\begin{array}{l}
x_{k+1}=A x_{k}+\left(B-\Delta\left(\epsilon_{k}\right)\right) u_{k-d_{1}}+\Delta\left(\epsilon_{k}\right) u_{k-d_{2}} \\
\forall d_{1}, d_{2} \in\left\{d_{\min }, \cdots, d_{\max }\right\}, \text { and } d_{1}>d_{2}
\end{array}\right.
$$

The nominal (delay-free and no packet loss) dynamics corresponding to $\epsilon_{k}=T_{s}$ are described by the following model:

$$
\begin{gathered}
\qquad x_{k+1}=A x_{k}+B u_{k} \\
\text { obtained for } \Delta\left(T_{s}\right)=B ; d_{2}=0 ; d_{1}=1 .
\end{gathered}
$$

First, assume that the PWA feedback controller (12), obtained for example from an explicit MPC design upon the nominal model (29) is used in practice for the control of the dynamics (28). The closed-loop system resulting from applying:

$$
\left\{\begin{array}{l}
u_{k-d_{1}}^{p w a}=F_{j} x_{k-d_{1}}+g_{j}, \text { for } x_{k-d_{1}} \in \mathcal{X}_{j} \\
u_{k-d_{2}}^{p w a}=F_{i} x_{k-d_{2}}+g_{i}, \text { for } x_{k-d_{2}} \in \mathcal{X}_{i}
\end{array}\right.
$$

in (28) will be:

$$
\left\{\begin{array}{c}
x_{k+1}=A x_{k}+\left(B-\Delta\left(\epsilon_{k}\right)\right)\left[F_{j} x_{k-d_{1}}+g_{j}\right]+ \\
\Delta\left(\epsilon_{k}\right)\left[F_{i} x_{k-d_{2}}+g_{i}\right]=A x_{k}+\left(B-\Delta\left(\epsilon_{k}\right)\right) F_{j} x_{k-d_{1}}+ \\
\Delta\left(\epsilon_{k}\right) F_{i} x_{k-d_{2}}+\left(B-\Delta\left(\epsilon_{k}\right)\right) g_{j}+\Delta\left(\epsilon_{k}\right) g_{i} . \\
\forall x_{k-d_{1}} \in \mathcal{X}_{j}, x_{k-d_{2}} \in \mathcal{X}_{i} ; \\
\forall d_{1}, d_{2} \in\left\{d_{\min }, \cdots, d_{\max }\right\} ; \text { and } d_{1}>d_{2} \\
\Delta\left(\epsilon_{k}\right) \in C o\left\{\Delta_{1}, \cdots, \Delta_{s}\right\}, \forall \epsilon_{k} \in \mathbb{R}_{\left[0, T_{s}\right]}
\end{array}\right.
$$

which is equivalent to:

$$
\left\{\begin{aligned}
x_{k+1}= & A x_{k}+\sum_{\lambda=0}^{d_{\max }} \psi_{\lambda}\left(\delta_{1}, \delta_{2}, \Delta\right) x_{k-\lambda}+\left(B-\Delta\left(\epsilon_{k}\right)\right) g_{j} \\
+ & \Delta\left(\epsilon_{k}\right) g_{i} . \\
& \forall d_{1}, d_{2} \in\left\{d_{\min }, \cdots, d_{\max }\right\}, \text { and } d_{1}>d_{2} ; \\
& \forall(i, j) \text { s.t } x_{k-d_{1}} \in \mathcal{X}_{j}, x_{k-d_{2}} \in \mathcal{X}_{i} ; \\
& \Delta\left(\epsilon_{k}\right) \in C o\left\{\Delta_{1}, \cdots, \Delta_{s}\right\}, \forall \epsilon_{k} \in \mathbb{R}_{\left[0, T_{s}\right]},
\end{aligned}\right.
$$

with:

$$
\psi_{\lambda}\left(\delta_{1}, \delta_{2}, \Delta\right)=\left\{\begin{array}{l}
0 \quad \text { if } \lambda<d_{\text {min }} \\
\left(B-\Delta\left(\epsilon_{k}\right)\right) F_{j} \delta_{1}(\lambda)+\Delta\left(\epsilon_{k}\right) F_{i} \delta_{2}(\lambda) \\
\quad \text { if } d_{\min } \leq \lambda \leq d_{\max },
\end{array}\right.
$$

$\delta_{1}$ and $\delta_{2}$ are two vectors of binary variables:

$$
\delta_{p}=\left[\delta_{p}\left(d_{\min }\right) \cdots \delta_{p}(i) \cdots \delta_{p}\left(d_{\max }\right)\right], \forall p \in\{1,2\} .
$$

These variables have constraints, and this should be handled when modeling the discrete-time dynamics:

$$
\sum_{i=d_{\min }}^{d_{\max }} \delta_{1}(i)=1, \text { and } \sum_{i=d_{\min }}^{d_{\max }} \delta_{2}(i)=1
$$

Next, the constraint $d_{1}>d_{2}$ can be written in terms of $\delta_{1}, \delta_{2}$ as follows:

$$
\sum_{i=d_{\min }}^{d_{\max }} i \delta_{1}(i)>\sum_{j=d_{\min }}^{d_{\max }} j \delta_{2}(j)
$$

Summarizing the remarks and observations above, we have the following result:

Theorem 3.4: For any continuous linear time-invariant (LTI) system in closed-loop with a PWA control law defined in (12) and affected by variable and possibly large input delays, there exists a switching linear model affected by polytopic uncertainty:

$$
\left\{\begin{array}{c}
x_{k+1}=A x_{k}+\sum_{\lambda=0}^{d_{\max }} \psi_{\lambda}\left(\delta_{1}, \delta_{2}, \Delta(\epsilon)\right) x_{k-\lambda}+\left(B-\Delta\left(\epsilon_{k}\right)\right) g_{j} \\
+\Delta\left(\epsilon_{k}\right) g_{i} . \\
\forall d_{1}>d_{2} \in\left\{d_{\min }, \cdots, d_{\max }\right\} ; \\
\forall(i, j) \text { s.t } x_{k-d_{1}} \in \mathcal{X}_{j}, x_{k-d_{2}} \in \mathcal{X}_{i} ; \\
\left(\delta_{1}, \delta_{2}\right) \in\left(\{0,1\}^{d_{\max }+1-d_{\min }}\right)^{2} ; \\
\Delta\left(\epsilon_{k}\right) \in \operatorname{Co}\left\{\Delta_{1}, \cdots, \Delta_{s}\right\}, \forall \epsilon_{k} \in \mathbb{R}_{\left[0, T_{s}\right]}
\end{array}\right.
$$

which takes into account the effect of the time-varying input delay.

Corollary 3.5: For a given state $x(t)=x\left(k T_{s}\right)$ and the PWA control function $u(t)$ defined in (12), let $\xi_{k}$ be the following extended state-space vector:

$$
\xi_{k}=\left[x_{k}^{T}, x_{k-1}^{T}, \cdots, x_{k-d_{\max }}^{T}\right]^{T} \in \mathbb{R}^{n \times\left(d_{\max }+1\right)}
$$

Then $x\left(t+T_{s}\right)$ can be obtained as follows:

$$
x\left(t_{k}+T_{s}\right)=\operatorname{Proj}_{x}\left\{F_{k} \xi_{k}+G_{k}\right\}
$$

where:

$$
F_{k}=\left[\begin{array}{ccccc}
A+\psi_{0} & \psi_{1} & \psi_{2} & \cdots & \psi_{d_{\max }} \\
I_{n} & 0_{n \times n} & 0_{n \times n} & \cdots & 0_{n \times n} \\
0_{n \times n} & \ddots & \cdots & \cdots & 0_{n \times n} \\
\vdots & \ddots & \ddots & \ldots & \vdots \\
0_{n \times n} & \cdots & 0_{n \times n} & I_{n} & 0_{n \times n}
\end{array}\right]
$$




$$
G_{k}=\left[\begin{array}{c}
\left(B-\Delta\left(\epsilon_{k}\right)\right) g_{j}+\Delta\left(\epsilon_{k}\right) g_{i} \\
0_{n \times 1} \\
\vdots \\
0_{n \times 1}
\end{array}\right]
$$

Proof: This is a direct consequence of the polytopic uncertainty embedding in [23] and the assumption made with respect to the piecewise affine structure of the control law.

To summarize, delay margin problem corresponds to the complete description of all possible delay values, denoted by $\left(d_{i n t}\right.$ or $\left.\mathcal{D}_{i n t}\right)$ for the intersample delay case, and by $\left(d_{\text {mult }}\right.$ or $\mathcal{D}_{\text {mult }}$ ) for the multisample case, such that the positive invariance of the set $\mathcal{X}$ (or alternatively $\underbrace{\mathcal{X} \times \cdots \times \mathcal{X}}_{d_{\max }+1}$ ), over which the PWA control law is defined, is guaranteed with respect to the corresponding closed-loop system.

\section{CONSTRUCTION OF THE DELAY MARGIN SET BASED ON THE POSITIVE INVARIANCE}

Assume that both $\mathcal{X}$ and the elements $\mathcal{X}_{i}$ defining the partition in (12) are bounded polyhedral sets. Then, the polytopes of interest can be described as the intersection of finite number of half spaces (referred to as the $\mathcal{H}$-representation):

$$
\begin{gathered}
\mathcal{X}=\left\{x \in \mathbb{R}^{n} \mid F x \leq f\right\}, F \in \mathbb{R}^{r \times n}, f \in \mathbb{R}^{r} \\
\mathcal{X}_{i}=\left\{x \in \mathbb{R}^{n} \mid F_{i} x \leq f_{i}\right\}, F_{i} \in \mathbb{R}^{r_{i} \times n}, f_{i} \in \mathbb{R}^{r_{i}}, \\
\text { for every } i \in \mathcal{I}_{N} . \\
\mathcal{X} \times \mathcal{X}=\left\{X \in \mathbb{R}^{2 n} \mid\left[\begin{array}{cc}
F & 0_{r \times n} \\
0_{r \times n} & F
\end{array}\right] X \leq\left[\begin{array}{l}
f \\
f
\end{array}\right]\right\} \\
\mathcal{X}_{i} \times \mathcal{X}_{j}=\left\{\begin{array}{c}
\left.X \in \mathbb{R}^{2 n} \mid\left[\begin{array}{cc}
F_{i} & 0_{r_{i} \times n} \\
0_{r_{j} \times n} & F_{j}
\end{array}\right] X \leq\left[\begin{array}{l}
f_{i} \\
f_{j}
\end{array}\right]\right\}, \\
\text { for every }(i, j) \in \mathcal{I}_{N}^{2} .
\end{array}\right.
\end{gathered}
$$

The polytopes defined above can also be described as the convex hull of finite point set (vertices) in $\mathbb{R}^{n}$ (referred to as the $\mathcal{V}$-representation). Let the vertices of the polytopes $\mathcal{X}$ and $\mathcal{X}_{i}$ be:

$$
\begin{gathered}
\mathcal{V}(\mathcal{X})=\left\{v_{1}, v_{2}, \cdots, v_{q}\right\}, \\
\mathcal{V}\left(\mathcal{X}_{i}\right)=\left\{w_{i 1}, w_{i 2}, \cdots, w_{i q_{i}}\right\}, \forall i \in \mathcal{I}_{N} .
\end{gathered}
$$

Then, the vertex representation of these polytopes is expressed using the convex hull operation as follows:

$$
\begin{gathered}
\mathcal{X}=\operatorname{Conv}\left\{v_{1}, v_{2}, \cdots, v_{q}\right\}, \\
\mathcal{X}_{i}=\operatorname{Conv}\left\{w_{i 1}, w_{i 2}, \cdots, w_{i q_{i}}\right\}, \forall i \in \mathcal{I}_{N} .
\end{gathered}
$$

For each region $\mathcal{X}_{i}$ of the partition of $\mathcal{X}$, the set containing its vertices is:

$$
\mathcal{W}_{i}=\mathcal{V}\left(\mathcal{X}_{i}\right), \forall i \in \mathcal{I}_{N} .
$$

Let $\mathcal{W}$ be the set of all vertices of all $\mathcal{X}_{i}$ with $i \in \mathcal{I}_{N}$ :

$$
\mathcal{W}=\bigcup_{i \in \mathcal{I}_{N}} \mathcal{V}\left(\mathcal{X}_{i}\right)
$$

Using only the non-redundant elements of $\mathcal{W}$, one can write:

$$
\mathcal{W}=\left\{w_{1}, w_{2}, \cdots, w_{p}\right\}, \operatorname{card}\{\mathcal{W}\}=p .
$$

The vertices of the polytope $\mathcal{X} \times \mathcal{X}$ are denoted by:

$$
\mathcal{V}(\mathcal{X} \times \mathcal{X})=\left\{\left(\begin{array}{c}
v_{i} \\
v_{j}
\end{array}\right) \in \mathbb{R}^{2 n}, \forall(i, j) \in \mathcal{I}_{q}^{2}\right\} .
$$

For each region $\mathcal{X}_{i} \times \mathcal{X}_{j}$ of the partition of $\mathcal{X} \times \mathcal{X}$, its set of vertices is:

$$
\begin{gathered}
\mathcal{W}_{i j}=\left[\mathcal{V}\left(\mathcal{X}_{i} \times \mathcal{X}_{j}\right)\right]=\mathcal{W}_{i} \times \mathcal{W}_{j}, \\
\mathcal{W}_{i j}=\left\{w_{i 1}, w_{i 2}, \cdots, w_{i q_{i}}\right\} \times\left\{w_{j 1}, w_{j 2}, \cdots, w_{j q_{j}}\right\} .
\end{gathered}
$$

Let $\mathcal{W}_{\mathcal{X} \times \mathcal{X}}$ be the set of all vertices of all $\mathcal{X}_{i} \times \mathcal{X}_{j}$ with the pairs $(i, j) \in \mathcal{I}_{N}^{2}$ :

$$
\mathcal{W}_{\mathcal{X} \times \mathcal{X}}=\bigcup_{(i, j) \in \mathcal{I}_{N}^{2}} \mathcal{V}\left(\mathcal{X}_{i} \times \mathcal{X}_{j}\right)
$$

It is worth to mention that there exists a close link between the elements of the two sets $\mathcal{W}$ and $\mathcal{W}_{\mathcal{X} \times \mathcal{X}}$. One can easily notice that:

$\mathcal{W}_{\mathcal{X} \times \mathcal{X}}=\left\{\left(\begin{array}{c}w_{i} \\ w_{j}\end{array}\right) \in \mathbb{R}^{2 n} \mid w_{i} \in \mathcal{W}, w_{j} \in \mathcal{W}, \forall(i, j) \in \mathcal{I}_{p}^{2}\right\}$

Based on the above notations, we define the following matrices obtained by storing as columns the non-redundant elements of the different sets of vertices using an arbitrary ordering:

$$
\begin{aligned}
& V=[\mathcal{V}(\mathcal{X})] \in \mathbb{R}^{n \times q}, \quad V_{\mathcal{X} \times \mathcal{X}}=[\mathcal{V}(\mathcal{X} \times \mathcal{X})] \in \mathbb{R}^{2 n \times q^{2}} \\
& V_{i}=\left[\mathcal{W}_{i}\right] \in \mathbb{R}^{n \times q_{i}}, \quad V_{i j}=\left[\mathcal{W}_{i j}\right] \in \mathbb{R}^{2 n \times\left(q_{i} \times q_{j}\right)} \\
& W=[\mathcal{W}] \in \mathbb{R}^{n \times p}, \quad W_{\mathcal{X} \times \mathcal{X}}=\left[\mathcal{W}_{\mathcal{X} \times \mathcal{X}}\right] \in \mathbb{R}^{2 n \times p^{2}}
\end{aligned}
$$

The image of the sets $\mathcal{W}_{i}, \mathcal{W}$ using the affine mapping (12) allows the construction of the matrices:

$$
\begin{aligned}
U_{i} & =\left[u^{p w a}\left(\mathcal{W}_{i}\right)\right] \in \mathbb{R}^{m \times q_{i}} \\
U & =\left[u^{p w a}(\mathcal{W})\right] \in \mathbb{R}^{m \times p} .
\end{aligned}
$$

respectively. Let $O_{p}^{k}$ be the $p \times p$ matrix whose all entries are equal to zero, except the $k^{t h}$ row, which is equal to $\mathbb{1}_{p}^{T}$.

\section{A. Intersample delay}

1) Delay margins in the extended state-space representation: The uncertainty in (8) is represented by the matrix $\Delta\left(\epsilon_{k}\right)$ satisfying $\Delta\left(\epsilon_{k}\right) \in \boldsymbol{\Delta}, k \in \mathbb{N}$, with:

$$
\boldsymbol{\Delta}=\left\{\Delta\left(\epsilon_{k}\right) \mid \epsilon_{k} \in\left[0, T_{s}\right]\right\}
$$

To characterize the delay margins we aim to use a simplicial over-approximation of the matrices $\Delta \in \mathbb{R}^{n \times m}$ in (60). Based on such an over-approximation of the matrix set $\Delta$, the system is embedded within a polytopic model with $s+1$ extreme realizations:

$$
\boldsymbol{\Delta} \in \operatorname{Conv}\left\{\Delta_{0}, \Delta_{1}, \cdots, \Delta_{s}\right\},
$$

any element of $\boldsymbol{\Delta}$ can be written as convex combinations of generators (corresponding to extreme realizations), i.e.:

$$
\forall \epsilon_{k} \in \mathbb{R}_{\left[0, T_{s}\right]}, \exists \alpha \in S_{s+1} \text { such that } \boldsymbol{\Delta}=\sum_{i=0}^{s} \alpha_{i} \Delta_{i} .
$$


Furthermore, with respect to the set $\mathcal{D}_{\text {int }} \subset \mathbb{R}_{\left[0, T_{s}\right]}$, we have:

$$
\forall \epsilon_{k} \in \mathcal{D}_{\text {int }}, \exists \alpha \in S_{s+1} \text { such that } \Delta=\sum_{i=0}^{s} \alpha_{i} \Delta_{i} .
$$

For a given $\xi_{k}$ in (15) such that $x_{k} \in \mathcal{X}_{i}$ and $x_{k-1} \in \mathcal{X}_{j}$, the feedback law is known i.e. $F_{i}, F_{j}, G_{i}$, and $G_{j}$ defining $\Phi_{i j}\left(\epsilon_{k}\right)$ and $\Gamma_{i j}\left(\epsilon_{k}\right)$ in (18) are known.

Proposition 4.1: For a given pair $(i, j) \in \mathcal{I}_{N}^{2}$, the matrix $\left[\Phi_{i j}\left(\epsilon_{k}\right) \Gamma_{i j}\left(\epsilon_{k}\right)\right]$ belongs to the polytopic set $\Omega$ :

$$
\Omega=\operatorname{Conv}\left\{\left[\Phi_{i j}^{1} \Gamma_{i j}^{1}\right], \cdots,\left[\Phi_{i j}^{s+1} \Gamma_{i j}^{s+1}\right]\right\}
$$

and there exists a vector $\alpha$ with non-negative scalars $\left\{\alpha_{0}, \cdots, \alpha_{s}\right\}$ such that $\alpha \in S_{s+1}$ satisfying:

$$
\left[\Phi_{i j}\left(\epsilon_{k}\right) \Gamma_{i j}\left(\epsilon_{k}\right)\right]=\sum_{l=0}^{s} \alpha_{l}\left[\Phi_{i j}^{l} \Gamma_{i j}^{l}\right]
$$

where:

$$
\begin{gathered}
\Phi_{i j}^{l}=\left[\begin{array}{cc}
A+\Delta_{l} F_{i} & \left(B-\Delta_{l}\right) F_{j} \\
I_{n} & 0_{n \times n}
\end{array}\right], \\
\Gamma_{i j}^{l}=\left[\begin{array}{c}
B g_{j}+\Delta_{l}\left(g_{i}-g_{j}\right) \\
0_{n \times 1}
\end{array}\right] .
\end{gathered}
$$

Proof: See Appendix A.

Theorem 4.2: Consider the uncertain piecewise affine system (18) defined over the polyhedral partition of $\mathcal{X} \times \mathcal{X}$. The delay margin is obtained in terms of $\alpha$ as:

$$
\mathcal{D}_{\text {int }}^{\alpha}=\left\{\operatorname{Proj}_{S_{s+1}} \mathcal{R}\right\} \cap \boldsymbol{\Delta}_{\alpha}
$$

where $\mathcal{R}$ and $\boldsymbol{\Delta}_{\alpha}$ are defined as:

$$
\begin{gathered}
\boldsymbol{\Delta}_{\alpha}=\left\{\alpha \in S_{s+1} \mid \forall \epsilon_{k} \in \mathbb{R}_{\left[0, T_{s}\right]} ; \Delta\left(\epsilon_{k}\right)=\sum_{i=0}^{s} \alpha_{i} \Delta_{i}\right\} \\
\mathcal{R}=\left\{(\alpha, \Gamma) \in \mathbb{R}_{+}^{s+1} \times \mathbb{R}_{+}^{q^{2} \times p^{2}} \mid \mathbb{1}^{T} \Gamma=\mathbb{1}^{T}\right. \\
\left.E+\sum_{l=0}^{l=s} \alpha_{l}\left[\begin{array}{cc}
\Delta_{l} & 0_{n \times m} \\
0_{n \times m} & 0_{n \times m}
\end{array}\right] H=V_{\mathcal{X} \times \mathcal{X}} \Gamma\right\}
\end{gathered}
$$

Where the matrices $E \in \mathbb{R}^{2 n \times p^{2}}, H \in \mathbb{R}^{2 m \times p^{2}}$ are defined in the proof.

Proof: See Appendix B.

2) Delay margins in the original state-space representation:

Theorem 4.3: Consider the uncertain piecewise affine systems (16) defined over the polyhedral partition of $\mathcal{X}$. The delay margin in the original state space representation is obtained in terms of $\alpha$ as follows:

$$
d_{\text {int }}^{\alpha}=\left\{\operatorname{Proj}_{S_{s+1}} \mathcal{T}\right\} \cap \boldsymbol{\Delta}_{\alpha}
$$

where $\boldsymbol{\Delta}_{\alpha}$ is defined in (68) and $\mathcal{T}$ is defined as:

$$
\begin{gathered}
\mathcal{T}=\left\{(\alpha, L) \in \mathbb{R}_{+}^{s+1} \times \mathbb{R}_{+}^{q \times p^{2}} \mid \mathbb{1}^{T} L=\mathbb{1}^{T},\right. \\
\left.E^{\prime}+\sum_{l=0}^{l=s} \alpha_{l} \Delta_{l} H^{\prime}=V L\right\}
\end{gathered}
$$

Where the matrices $E^{\prime} \in \mathbb{R}^{n \times p^{2}}, H^{\prime} \in \mathbb{R}^{m \times p^{2}}$ are given by:

$$
\begin{gathered}
E^{\prime}=\left[\begin{array}{lll}
A W O_{p}^{1}+B U & \cdots & A W O_{p}^{p}+B U
\end{array}\right], \\
H^{\prime}=\left[\begin{array}{lll}
U O_{p}^{1}-U & \cdots & U O_{p}^{p}-U
\end{array}\right] .
\end{gathered}
$$

Proof: See Appendix C.

3) Relationship between delay margins: The link between the two representations and their invariant sets has received a unifying characterization via set factorization in [17]. This relationship is formally stated in the next theorem and for the sake of brevity, the proof is omitted. For more details the reader is referred to [17] and the references therein.

Theorem 4.4: The dynamical system (16) admits a convex $\mathcal{D}$-invariant set if and only if there exists an invariant set for the system (18) which admits a regular ordered factorization.

Even if the existence of an invariant set with respect to the extended dynamics (18) represents only a necessary condition for the existence of a $\mathcal{D}$-invariant set with respect to (16), the following proposition holds in the analysis of delay margins.

Proposition 4.5: The delay margin sets $d_{\text {int }}$ and $\mathcal{D}_{\text {int }}$ are equivalent.

Proof: See Appendix D.

Note that the same result holds in the multisample case. Thus, we will restrict ourselves to the study of delay margins in the original state space representation $d_{\text {mult }}$.

\section{B. Large delays and Packet dropouts}

Starting from a PWA control law design constructed upon a nominal delay-free model, we derive a generalized solution to the delay margin problem. We provide a constructive method to find the delay margins i.e. the set of all time-varying delays, possibly larger than one sampling period where packets loss may occur, for which the set $\mathcal{X}$ is positively invariant with respect to the corresponding closed-loop dynamics. The discretemodel of interest, describing the control system including delays and packet dropouts, has been presented in (37). Note that since the delay margins were pointed out to be equivalent in the original and the augmented state-space representations, we will be exclusively interested in finding all delay values for which the $\mathcal{D}$-invariance of the closed-loop system (37) is guaranteed. The delay margin in the extended state-space representation ensuring the invariance of the set

$$
\mathcal{P}=\underbrace{\mathcal{X} \times \cdots \times \mathcal{X}}_{d_{\max }+1}
$$

with respect to $\xi_{k+1}=F_{k} \xi_{k}+G_{k}$ (Corollary 3.5) being exactly the same.

Theorem 4.6: Consider the closed-loop system described by the delay difference equation (37) defined over the polyhedral partition of $\mathcal{X}$. For a fixed value of the pair $d_{1}, d_{2} \in$ $\left\{d_{\min }, \cdots, d_{\max }\right\}$, the intersample delay margin $d_{* i n t}$ is obtained as follows:

$$
d_{* i n t}^{\alpha}=\left\{\operatorname{Proj}_{S_{s+1}} \mathcal{T}^{\prime}\right\} \cap \boldsymbol{\Delta}_{\alpha}
$$


where $\boldsymbol{\Delta}_{\alpha}$ is defined in (68) and $\mathcal{T}^{\prime}$ is defined as:

$$
\begin{gathered}
\mathcal{T}^{\prime}=\left\{(\alpha, L) \in \mathbb{R}_{+}^{s+1} \times \mathbb{R}_{+}^{q \times p^{3}} \mid \mathbb{1}^{T} L=\mathbb{1}^{T},\right. \\
\left.E^{\prime \prime}+\sum_{l=0}^{l=s} \alpha_{l} \Delta_{l} H^{\prime \prime}=V L\right\}
\end{gathered}
$$

where the matrices $E^{\prime \prime} \in \mathbb{R}^{n \times p^{3}}$ and $H^{\prime \prime} \in \mathbb{R}^{m \times p^{3}}$ are defined as follows:

$$
E^{\prime \prime}=A\left[\mathbb{1}_{p}^{T} \otimes W O_{p}^{1} \cdots \mathbb{1}_{p}^{T} \otimes W O_{p}^{p}\right]+B\left[\mathbb{1}_{p^{2}}^{T} \otimes U\right]
$$

and

$$
H^{\prime \prime}=\mathbb{1}_{p}^{T} \otimes\left[U O_{p}^{1}-U \cdots U O_{p}^{p}-U\right] .
$$

Proof: See Appendix E.

Proposition 4.7: The following claims hold when dealing with delay margins in the presence of large delays:

i) $\tau_{k}^{*}=0$ belongs to the multisample delay margin $d_{\text {mult }}$ if the nominal system is positive invariant in closed-loop with the PWA control law defined over $\mathcal{X}$.

ii) If the intersample delay margin for a fixed value of the pair $d_{1}, d_{2} \in\left\{d_{\min }, \cdots, d_{\max }\right\}$ is not empty $\left(d_{* i n t} \neq\right.$ $\emptyset)$, then $\tau_{i}^{*}=d_{i} T_{s}, \forall d_{i} \in\left\{1, \cdots, d_{\max }-1\right\}$ belong to $d_{\text {mult }}$. Furthermore, $d_{* i n t} \subset\left[0, T_{s}\right]$ in this case.

iii) If $\left[d_{i} T_{s}, d_{i} T_{s}+\tau_{M}\right] \subset d_{\text {mult }}$ for a given $d_{i} \in\left\{d_{\min }, \cdots, d_{\max }-1\right\}$, then the same inclusions $\left[d_{j} T_{s}, d_{j} T_{s}+\tau_{M}\right] \subset d_{\text {mult }}$ hold for all $d_{j} \in\left\{1, \cdots, d_{\max }-1\right\}$.

Proof:

i) The PWA controller has been designed upon the nominal (delay-free) model. If Assumption 3.1 holds, then the invariance of the set $\mathcal{X}$ with respect to the closed-loop system will be guaranteed, and $\{0\} \in d_{\text {int }} \subset d_{\text {mult }}$.

ii) Suppose that $d_{* i n t} \neq \emptyset$, then the following $\mathcal{D}$-invariance condition holds:

$$
A \mathcal{X} \oplus \Delta F_{i} \mathcal{X} \oplus(B-\Delta) F_{j} \mathcal{X} \oplus(B-\Delta) g_{j} \oplus \Delta g_{j} \subseteq \mathcal{X}
$$

for $(i, j) \in \mathcal{I}_{N}^{2}$, such that $x_{k-d_{1}} \in \mathcal{X}$ and $x_{k-d_{2}} \in \mathcal{X}$, $\forall \tau_{k}^{*} \in d_{\text {mult }}$. Choosing, $i=j=k$, (76) yields:

$$
A \mathcal{X} \oplus(B-\Delta+\Delta) F_{k} \mathcal{X} \oplus(B-\Delta+\Delta) g_{k} \subseteq
$$$$
A \mathcal{X} \oplus \Delta F_{i} \mathcal{X} \oplus(B-\Delta) F_{j} \mathcal{X} \oplus(B-\Delta) g_{j} \oplus \Delta g_{j} \subseteq \mathcal{X}
$$

or equivalently:

$$
A \mathcal{X} \oplus B F_{k} \mathcal{X} \oplus B g_{k} \subseteq \mathcal{X}, \forall k \in \mathcal{I}_{N}
$$

Since (78) corresponds to the $\mathcal{D}$-invariance condition for a delay $\tau_{k}^{*}=d_{k} T_{s}, \forall d_{k} \in\left\{1, \cdots, d_{\max }-1\right\}$, it follows that the delay margin covers $\tau_{k}^{*}=d_{k} T_{s} \in d_{\text {mult }}, d_{k} \in$ $\left\{1, \cdots, d_{\max }-1\right\}$.

Notice from the Minkowski sum properties that the following inclusion:

$$
\left(A+B F_{i}\right) \mathcal{X} \oplus B_{i} g_{i} \subseteq \mathcal{X}, \forall i \in \mathcal{I}_{N}
$$

which corresponds to the invariance for $\tau_{k}^{*}=0$, does not necessarily imply:

$$
A \mathcal{X} \oplus B F_{i} \mathcal{X} \oplus B_{i} g_{i} \subseteq \mathcal{X}, \forall i \in \mathcal{I}_{N}
$$

In other words, $\tau_{i}^{*}=d_{i} T_{s}$, where $d_{i} \in$ $\left\{1, \cdots, d_{\max }-1\right\}$ do not necessarily belong to the multisample delay margin $d_{\text {mult }}$ if $\{0\} \in d_{\text {mult }}$.

iii) The proof of this statement follows directly from the delay independent property of $\mathcal{D}$-invariance (see Proposition 2.6).

Corollary 4.8: The delay margin $d_{\text {mult }}$ can be written as a union of sets as follows:

$$
d_{\text {mult }}=d_{\text {int }} \bigcup\left(T_{s} \oplus d_{* i n t}\right) \bigcup \cdots \bigcup\left(\left(d_{\text {max }}-1\right) T_{s} \oplus d_{* i n t}\right) .
$$

where $d_{* i n t}$ is a subset of the intersample delay margin, $d_{i n t}$ and is obtained from (72).

Proof: The proof follows from the multisample delay margin properties $d_{\text {mult }}$ in Proposition 4.7. First we observe that globally the delay margin is a union of the intersample delay $d_{\text {int }}$ and the intersample delay margins for all possible combinations of $d_{1}, d_{2} \in\left\{d_{\min }, \cdots, d_{\max }\right\}$ with $d_{1}>d_{2}$. Secondly by exploiting the second property, the inclusion of one integer multiple of the sampling time in the delay margin, any integer value will be also included. Finally, the property iii) in Proposition 4.7, the subintervals of delay will be replicated at each sampling interval leading to the form presented in the statement.

\section{ILluStRATIVE EXAMPLE}

Consider the following unstable dynamical system:

$$
\left\{\begin{array}{l}
\dot{x}(t)=\left[\begin{array}{cc}
1.1 & -0.1 \\
1 & 0
\end{array}\right] x(t)+\left[\begin{array}{l}
1 \\
0
\end{array}\right] u(t-h) \\
y(t)=\left[\begin{array}{ll}
1 & 0
\end{array}\right] x(t) .
\end{array}\right.
$$

with $h \in[0,0.1]$. A discrete model is obtained, using (6)-(8) with a sampling period $T_{s}=0.1$, and the uncertainty $0<$ $\epsilon_{k} \leq 0.1$. The uncertainty matrix $\Delta\left(\epsilon_{k}\right)$ has been embedded within a polytopic model with the following 3 vertices:

$$
\Delta \in \operatorname{Conv}\left\{\left[\begin{array}{l}
0 \\
0
\end{array}\right],\left[\begin{array}{c}
0.0999 \\
-0.0006
\end{array}\right],\left[\begin{array}{l}
0.1057 \\
0.0057
\end{array}\right]\right\}
$$

Then, an explicit MPC has been designed for the nominal model (delay-free, $h=0$ ), with a prediction horizon $N=7$, in the presence of input and output constraints:

$$
\begin{aligned}
& -5 \leq u_{k} \leq 5 \\
& -5 \leq y_{k} \leq 5
\end{aligned}
$$

The partition of the obtained PWA control law as well as the resulting over-approximation of the uncertainty are shown in Figure 1.

The delay margin $d_{m}^{\alpha}$ has been computed using (72). Its projection on the plane $\left(\alpha_{0}, \alpha_{1}\right)$ is shown in Figure 2. The red set and the curved black line represent the sets $\mathcal{T}$ and $\Delta_{\alpha}$ defined in (73) and (68), respectively, both projected on $S_{2}$. Finally the delay margin is obtained $d_{m}=[0.0972 ; 0.1]$, which corresponds to a delay variation $\tau_{k} \in[0 ; 0.0028]$.

Note that some close links between the delay margins and the number of regions in the partition of the PWA control law exist. The number of regions being directly related to the choice of the prediction horizon, one can notice that the 

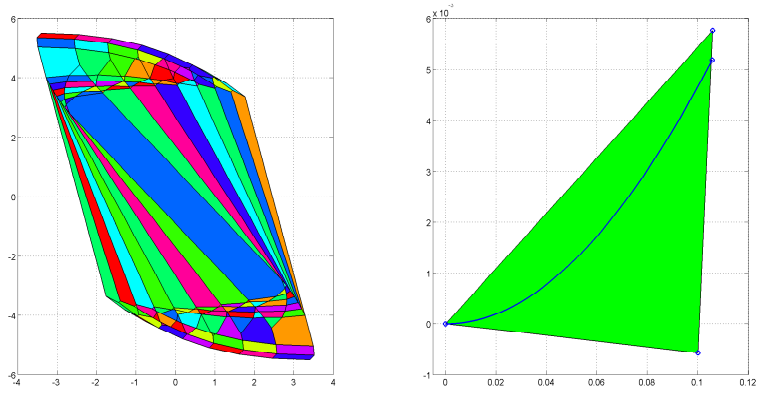

Fig. 1. Partition of the PWA control law (left), illustration of the embedding of $\Delta\left(\epsilon_{k}\right)$ by a simplex (right)

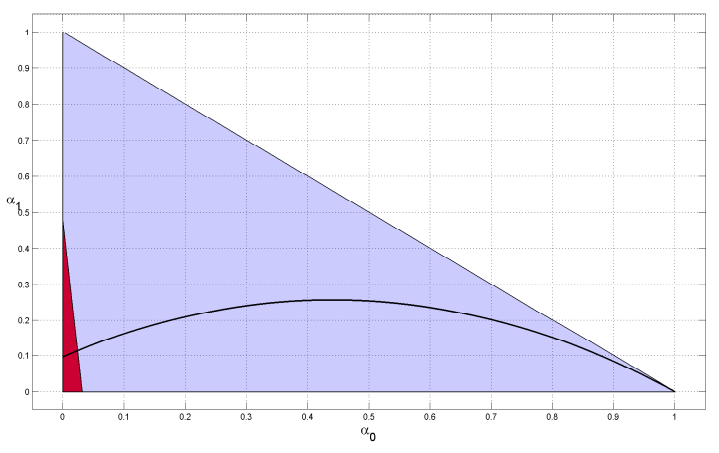

Fig. 2. Delay margin $d_{m}^{\alpha}$ projected on the plane $\left(\alpha_{0}, \alpha_{1}\right)$

delay margin is larger when the prediction horizon is small. Delay margins for different PWA control laws obtained using different prediction horizons are shown in Table I. The global delay margin is reduced to these intervals as long as the system is open-loop unstable and the $\mathcal{D}$-invariance can not be achieved. This is a natural consequence of the fact that, the $\mathcal{D}$-invariance is related to a delay independent stability.

TABLE I

DELAY MARGINS AND THE PREDICTION HORIZON

\begin{tabular}{|c|c|c|c|c|c|}
\hline$N$ & 2 & 3 & 4 & 5 & 6 \\
\hline$\tau_{k} \times 10^{3}$ & {$[0 ; 5.6]$} & {$[0 ; 4.9]$} & {$[0 ; 4.2]$} & {$[0 ; 3.7]$} & {$[0 ; 3.2]$} \\
\hline
\end{tabular}

\section{CONCLUSION}

In discrete-time modeling framework, the analysis of linear systems affected by uncertain time-varying input delays passes through the characterization of uncertain delay difference equations depending on a parameter varying matrix. Many different techniques exist in the literature aiming at constructing an embedding for the uncertainty, and leading to classical polytopic models.

In the present work, we addressed an inverse problem, offering a measure of the delay margin of positive invariance for a closed-loop PWA system in the original (related to $\mathcal{D}$ invariance) and the extended state-space representations for both small and large delays. The result presented in this paper gives a way to tackle the delay margin problem of a nominal PWA control law which can be seen as a relevant issue from the robustness analysis point of view in both feedback communication channels and variable computation-time for real-time optimization-based control.

\section{APPENDIX A}

\section{Proof of Proposition 4.1}

Since $\Delta$ lies inside a convex set, any matrix $\Phi_{i j}\left(\epsilon_{k}\right)$ for a given $\epsilon_{k} \in \mathbb{R}_{\left[0, T_{s}\right]}$ can also be written as convex combination of extreme realizations:

$$
\begin{aligned}
& \forall \epsilon_{k} \in \mathbb{R}_{\left[0, T_{s}\right]}, \exists \beta \in S_{K+1} \text { such that: } \\
\Phi_{i j}\left(\epsilon_{k}\right)= & {\left[\begin{array}{cc}
A+\Delta\left(\epsilon_{k}\right) F_{i} & \left(B-\Delta\left(\epsilon_{k}\right)\right) F_{j} \\
I_{n} & 0_{n \times n}
\end{array}\right]=\sum_{l=0}^{K} \beta_{l} \Phi_{i j}^{l}, }
\end{aligned}
$$

where:

$$
\begin{aligned}
& \Phi_{i j}^{l}=\left[\begin{array}{cc}
A+\Delta_{l} F_{i} & \left(B-\Delta_{l}\right) F_{j} \\
I_{n} & 0_{n \times n}
\end{array}\right] \\
&=\left[\begin{array}{cc}
A & B F_{j} \\
I_{n} & 0_{n \times n}
\end{array}\right]+\left[\begin{array}{cc}
\Delta_{l} & 0_{n \times m} \\
0_{n \times m} & 0_{n \times m}
\end{array}\right]\left[\begin{array}{cc}
F_{i} & -F_{j} \\
0_{m \times n} & 0_{m \times n}
\end{array}\right] .
\end{aligned}
$$

Same procedure can be applied for $\Gamma_{i j}\left(\epsilon_{k}\right)$ :

$$
\forall \epsilon_{k} \in \mathbb{R}_{\left[0, T_{s}\right]}, \exists \lambda \in S_{K+1} \text { such that: }
$$

$$
\Gamma_{i j}\left(\epsilon_{k}\right)=\left[\begin{array}{c}
B g_{j}+\Delta\left(\epsilon_{k}\right)\left(g_{i}-g_{j}\right) \\
0_{n \times 1}
\end{array}\right]=\sum_{l=0}^{K} \lambda_{l} \Gamma_{i j}^{l}
$$

where:

$$
\begin{gathered}
\Gamma_{i j}^{l}=\left[\begin{array}{cc}
B g_{j}+\Delta_{l}\left(g_{i}-g_{j}\right) & 0_{n \times 1}
\end{array}\right] \\
=\left[\begin{array}{c}
B g_{j} \\
0_{n \times 1}
\end{array}\right]+\left[\begin{array}{cc}
\Delta_{l} & 0_{n \times m} \\
0_{n \times m} & 0_{n \times m}
\end{array}\right]\left[\begin{array}{c}
g_{i}-g_{j} \\
0_{m \times 1}
\end{array}\right] .
\end{gathered}
$$

However, for a given $\epsilon_{k} \in \mathbb{R}_{\left[0, T_{s}\right]}$ and $(i, j) \in \mathcal{I}_{N}^{2}$, by selecting $\beta_{l}=\lambda_{l}:=\alpha_{l}, \forall l \in \mathbb{Z}_{[0, K]}$, one concludes that (64) holds with $\Phi_{i j}^{l}$ and $\Gamma_{i j}^{l}$ described in (83) and (85) respectively.

\section{APPENDIX B}

\section{PROOF OF THEOREM 4.2}

The positive invariance of the set $\mathcal{X} \times \mathcal{X}$ with respect to the time-varying dynamical system (18) is represented by a set-wise relation:

$$
\begin{gathered}
\forall \epsilon_{k} \in \mathcal{D}_{m} \subset \mathbb{R}_{\left[0, T_{s}\right]}, \text { and } \forall \xi_{k} \in \mathcal{X}_{i} \times \mathcal{X}_{j},(i, j) \in \mathcal{I}_{N}^{2}: \\
\Phi_{i j}\left(\epsilon_{k}\right) \xi_{k}+\Gamma_{i j}\left(\epsilon_{k}\right) \in \mathcal{X} \times \mathcal{X},
\end{gathered}
$$

which is equivalent to:

$$
\begin{gathered}
\forall \epsilon_{k} \in \mathcal{D}_{m} \subset \mathbb{R}_{\left[0, T_{s}\right]}, \text { and } \forall \xi_{k} \in \mathcal{X}_{i} \times \mathcal{X}_{j},(i, j) \in \mathcal{I}_{N}^{2}, \\
\exists \alpha \in S_{s+1} \text { such that } \sum_{l=0}^{s} \alpha_{l}\left[\Phi_{i j}^{l} \xi_{k}+\Gamma_{i j}^{l}\right] \in \mathcal{X} \times \mathcal{X} .
\end{gathered}
$$

By substituting (83) and (85) in equation (87), we obtain:

$\sum_{l=0}^{s} \alpha_{l}\left[\left(\left[\begin{array}{cc}A & B F_{j} \\ I_{n} & 0_{n \times n}\end{array}\right]+\left[\begin{array}{cc}\Delta_{l} & 0_{n \times m} \\ 0_{n \times m} & 0_{n \times m}\end{array}\right]\left[\begin{array}{cc}F_{i} & -F_{j} \\ 0_{m \times n} & 0_{m \times n}\end{array}\right]\right)\right.$ 


$$
\begin{gathered}
\left.\xi_{k}+\left[\begin{array}{c}
B g_{j} \\
0_{n \times 1}
\end{array}\right]+\left[\begin{array}{cc}
\Delta_{l} & 0_{n \times m} \\
0_{n \times m} & 0_{n \times m}
\end{array}\right]\left[\begin{array}{c}
g_{i}-g_{j} \\
0_{m \times 1}
\end{array}\right]\right] \in \mathcal{X} \times \mathcal{X} . \\
{\left[\begin{array}{cc}
A & B F_{j} \\
I_{n} & 0_{n \times n}
\end{array}\right] \xi_{k}+\left[\begin{array}{c}
B g_{j} \\
0_{n \times 1}
\end{array}\right]+\sum_{l=0}^{s} \alpha_{l}\left[\begin{array}{cc}
\Delta_{l} & 0_{n \times m} \\
0_{n \times m} & 0_{n \times m}
\end{array}\right]} \\
\left(\left[\begin{array}{cc}
F_{i} & -F_{j} \\
0_{m \times n} & 0_{m \times n}
\end{array}\right] \xi_{k}+\left[\begin{array}{c}
g_{i}-g_{j} \\
0_{m \times 1}
\end{array}\right]\right) \in \mathcal{X} \times \mathcal{X} .
\end{gathered}
$$

By expressing the extended state vector $\xi_{k} \in \mathcal{X}_{i} \times \mathcal{X}_{j}$ as a convex combinations of the vertices of $\mathcal{X}_{i} \times \mathcal{X}_{j}$ which is known to be polyehdral set, we obtain:

$$
\xi_{k}=\sum_{z=1}^{q_{i} \times q_{j}} \beta_{z} w_{i j}^{z} \text { for } \beta \in S_{q_{i} \times q_{j}} .
$$

It follows that equation (89) is equivalent with:

$$
\begin{array}{r}
{\left[\begin{array}{cc}
A & B F_{j} \\
I_{n} & 0_{n \times n}
\end{array}\right] \sum_{z=1}^{q_{i} \times q_{j}} \beta_{z} w_{i j}^{z}+\left[\begin{array}{c}
B g_{j} \\
0_{n \times 1}
\end{array}\right]+\sum_{l=0}^{s} \alpha_{l}\left[\begin{array}{cc}
\Delta_{l} & 0_{n \times m} \\
0_{n \times m} & 0_{n \times m}
\end{array}\right]} \\
\left(\left[\begin{array}{cc}
F_{i} & -F_{j} \\
0_{m \times n} & 0_{m \times n}
\end{array}\right] \sum_{z=1}^{q_{i} \times q_{j}} \beta_{z} w_{i j}^{z}+\left[\begin{array}{c}
g_{i}-g_{j} \\
0_{m \times 1}
\end{array}\right]\right) \in \mathcal{X} \times \mathcal{X} .
\end{array}
$$

For a given vertex in $w_{i j}^{z}$, i.e. $z \in \mathbb{Z}_{\left[1, q_{i} \times q_{j}\right]},(i, j) \in \mathcal{I}_{N}^{2}$, we have:

$$
\begin{array}{r}
{\left[\begin{array}{cc}
A & B F_{j} \\
I_{n} & 0_{n \times n}
\end{array}\right] w_{i j}^{z}+\left[\begin{array}{c}
B g_{j} \\
0_{n \times 1}
\end{array}\right]+\sum_{l=0}^{s} \alpha_{l}\left[\begin{array}{cc}
\Delta_{l} & 0_{n \times m} \\
0_{n \times m} & 0_{n \times m}
\end{array}\right]} \\
\left(\left[\begin{array}{cc}
F_{i} & -F_{j} \\
0_{m \times n} & 0_{m \times n}
\end{array}\right] w_{i j}^{z}+\left[\begin{array}{c}
g_{i}-g_{j} \\
0_{m \times 1}
\end{array}\right]\right) \in \mathcal{X} \times \mathcal{X} .
\end{array}
$$

We describe the inclusion (92) explicitly since it is equivalent with the existence of a vector $y_{i j}^{z} \in \mathcal{X} \times \mathcal{X}$ such that:

$$
\begin{array}{r}
{\left[\begin{array}{cc}
A & B F_{j} \\
I_{n} & 0_{n \times n}
\end{array}\right] w_{i j}^{z}+\left[\begin{array}{c}
B g_{j} \\
0_{n \times 1}
\end{array}\right]+\sum_{l=0}^{s} \alpha_{l}\left[\begin{array}{cc}
\Delta_{l} & 0_{n \times m} \\
0_{n \times m} & 0_{n \times m}
\end{array}\right]} \\
\left(\left[\begin{array}{cc}
F_{i} & -F_{j} \\
0_{m \times n} & 0_{m \times n}
\end{array}\right] w_{i j}^{z}+\left[\begin{array}{c}
g_{i}-g_{j} \\
0_{m \times 1}
\end{array}\right]\right)=y_{i j}^{z},
\end{array}
$$

where the vector $y_{i j}^{z}$ can be expressed as:

$$
y_{i j}^{z}=V_{\mathcal{X} \times \mathcal{X}} \gamma_{i j}^{z} \text { such that } \gamma_{i j}^{z} \in S_{q^{2}} .
$$

By replacing equations (94) in (93), $\forall(i, j) \in \mathcal{I}_{N}^{2}$ and $z \in$ $\mathbb{Z}_{\left[1, q_{i} \times q_{j}\right]}$, we obtain:

$$
\begin{gathered}
{\left[\begin{array}{cc}
A & B F_{j} \\
I_{n} & 0_{n \times n}
\end{array}\right] w_{i j}^{z}+\left[\begin{array}{c}
B g_{j} \\
0_{n \times 1}
\end{array}\right]+\sum_{l=0}^{s} \alpha_{l}\left[\begin{array}{cc}
\Delta_{l} & 0_{n \times m} \\
0_{n \times m} & 0_{n \times m}
\end{array}\right]} \\
\left(\left[\begin{array}{cc}
F_{i} & -F_{j} \\
0_{m \times n} & 0_{m \times n}
\end{array}\right] w_{i j}^{z}+\left[\begin{array}{c}
g_{i}-g_{j} \\
0_{m \times 1}
\end{array}\right]\right)=V_{\mathcal{X} \times \mathcal{X} \gamma_{i j}^{z},}
\end{gathered}
$$

or, in other words, equation (95) holds for all non redundant vertices of $\mathcal{X}_{i} \times \mathcal{X}_{j}, \forall(i, j) \in \mathcal{I}_{N}^{2}$, which means that it holds for all the columns of the matrix $W_{\mathcal{X} \times \mathcal{X}}$ defined in (57). Exploiting the piecewise affine mapping (12) of the elements of $\mathcal{W}_{\mathcal{X} \times \mathcal{X}}$, a matrix formulation can be obtained by the concatenation of the vectors:

$$
\left[\begin{array}{cc}
A & B F_{j} \\
I_{n} & 0_{n \times n}
\end{array}\right] w_{i j}^{z}+\left[\begin{array}{c}
B g_{j} \\
0_{n \times 1}
\end{array}\right]
$$

and, similarly of the vectors:

$$
\left[\begin{array}{cc}
F_{i} & -F_{j} \\
0_{m \times n} & 0_{m \times n}
\end{array}\right] w_{i j}^{z}+\left[\begin{array}{c}
g_{i}-g_{j} \\
0_{m \times 1}
\end{array}\right]
$$

in (95). Indeed, the collection of vectors:

$$
\begin{gathered}
{\left[\begin{array}{cc}
A & B F_{j} \\
I_{n} & 0_{n \times n}
\end{array}\right] w_{i j}^{z}+\left[\begin{array}{c}
B g_{j} \\
0_{n \times 1}
\end{array}\right], w_{i j}^{z} \in \mathcal{W}_{\mathcal{X} \times \mathcal{X}} \rightarrow} \\
{\left[\begin{array}{cc}
A & 0_{n \times n} \\
I_{n} & 0_{n \times n}
\end{array}\right] W_{\mathcal{X} \times \mathcal{X}}+\left[\begin{array}{c}
B U \\
0_{n \times p}
\end{array}\right]\left[\begin{array}{lll}
I_{p} & \cdots & I_{p}
\end{array}\right] .}
\end{gathered}
$$

Next, the same procedure can be applied for (97):

$$
\begin{gathered}
{\left[\begin{array}{cc}
F_{i} & -F_{j} \\
0_{m \times n} & 0_{m \times n}
\end{array}\right] w_{i j}^{z}+\left[\begin{array}{c}
g_{i}-g_{j} \\
0_{m \times 1}
\end{array}\right], w_{i j}^{z} \in \mathcal{W}_{\mathcal{X} \times \mathcal{X}} \rightarrow} \\
{\left[\begin{array}{cccc}
U O_{p}^{1}-U & U O_{p}^{2}-U & \cdots & U O_{p}^{p}-U \\
0_{m \times p} & 0_{m \times p} & \cdots & 0_{m \times p}
\end{array}\right],}
\end{gathered}
$$

and

$$
\left\{\begin{array}{l}
V_{\mathcal{X} \times \mathcal{X}} \gamma_{i j}^{z} \\
w_{i j}^{z} \in \mathcal{W}_{\mathcal{X} \times \mathcal{X}}
\end{array}=V_{\mathcal{X} \times \mathcal{X}} \Gamma\right.
$$

with the condition that each column of $\Gamma$ is restricted to $S_{q^{2}}$. This can be expressed as linear constraints on the columns of $\Gamma$ as follows:

$$
\mathbb{1}^{T} \Gamma=\mathbb{1}^{T}, \quad \Gamma \in \mathbb{R}_{+}^{q^{2} \times p^{2}} .
$$

Finally, equation (95) leads to the matrix formulation:

$$
\underbrace{\left[\begin{array}{cc}
A & 0_{n \times n} \\
I_{n} & 0_{n \times n}
\end{array}\right] W_{\mathcal{X} \times \mathcal{X}}+\left[\begin{array}{c}
B U \\
0_{n \times p}
\end{array}\right]\left[\begin{array}{lll}
I_{p} & \cdots & I_{p}
\end{array}\right]}_{E}+
$$

$$
\begin{gathered}
\sum_{l=0}^{l=s} \alpha_{l}\left[\begin{array}{cc}
\Delta_{l} & 0_{n \times m} \\
0_{n \times m} & 0_{n \times m}
\end{array}\right] \underbrace{\left[\begin{array}{ccc}
U O_{p}^{1}-U & \cdots & U O_{p}^{p}-U \\
0_{m \times p} & \cdots & 0_{m \times p}
\end{array}\right]}_{H} \\
=V_{\mathcal{X} \times \mathcal{X} \Gamma}, \\
E+\sum_{l=0}^{l=s} \alpha_{l}\left[\begin{array}{cc}
\Delta_{l} & 0_{n \times m} \\
0_{n \times m} & 0_{n \times m}
\end{array}\right] H=V_{\mathcal{X} \times \mathcal{X} \Gamma .}
\end{gathered}
$$

Since the parametric uncertainty corresponds to the values of $\alpha$ in (68), $\left(\Delta\left(\epsilon_{k}\right)\right.$ does not take all values in the embedding), the delay margin is obtained as (67) in terms of $\alpha$. The proof is complete noticing that the two sets $\mathcal{D}_{m}^{\alpha}$ and $\mathcal{D}_{m}$ are isomorphic.

\section{APPENDIX C}

\section{PROOF OF THE THEOREM 4.3}

The $\mathcal{D}$-invariance of the set $\mathcal{X}$ with respect to the original time-varying dynamical system (16) is represented by a set wise relation $\forall \epsilon_{k} \in d_{m}$ :

$$
\begin{gathered}
\left(A+\Delta\left(\epsilon_{k}\right) F_{i}\right) x_{k}+\left(B-\Delta\left(\epsilon_{k}\right)\right) F_{j} x_{k-1}+B g_{j}+ \\
\Delta\left(\epsilon_{k}\right)\left(g_{i}-g_{j}\right) \in \mathcal{X}, \\
\forall(i, j) \in \mathcal{I}_{N}^{2} \text { s.t } x_{k} \in \mathcal{X}_{i} \text { and } x_{k-1} \in \mathcal{X}_{j},
\end{gathered}
$$


or, equivalently:

$$
\begin{gathered}
A x_{k}+B F_{j} x_{k-1}+B g_{j}+\Delta\left(\epsilon_{k}\right)\left[\left(F_{i} x_{k}+g_{i}\right)\right. \\
\left.-\left(F_{j} x_{k-1}+g_{j}\right)\right] \in \mathcal{X}, \\
\forall(i, j) \in \mathcal{I}_{N}^{2} \text { s.t } x_{k} \in \mathcal{X}_{i} \text { and } x_{k-1} \in \mathcal{X}_{j},
\end{gathered}
$$

which is equivalent, exploiting the convex combinations of extreme realizations of $\Delta\left(\epsilon_{k}\right)$, to:

$$
\begin{gathered}
A x_{k}+B F_{j} x_{k-1}+B g_{j}+\sum_{l=0}^{K} \alpha_{l} \Delta_{l}\left[\left(F_{i} x_{k}+g_{i}\right)\right. \\
\left.-\left(F_{j} x_{k-1}+g_{j}\right)\right] \in \mathcal{X}, \\
\forall(i, j) \in \mathcal{I}_{N}^{2} \text { s.t } x_{k} \in \mathcal{X}_{i} \text { and } x_{k-1} \in \mathcal{X}_{j},
\end{gathered}
$$

with $\alpha_{l}, l \in \mathbb{Z}_{[0, K]}$ being the elements of a vector $\alpha \in S_{K+1}$. By expressing the state vectors $x_{k} \in \mathcal{X}_{i}$ and $x_{k-1} \in \mathcal{X}_{j}$ as:

$$
\begin{gathered}
x_{k}=\sum_{l=1}^{q_{i}} \lambda_{l} w_{i l} \text { for } \lambda \in S_{q_{i}}, \\
x_{k-1}=\sum_{l^{\prime}=1}^{q_{j}} \lambda_{l^{\prime}}^{\prime} w_{j l^{\prime}} \text { for } \lambda^{\prime} \in S_{q_{j}} .
\end{gathered}
$$

Then, for a given pair of vertices in $w_{i l}, w_{j l^{\prime}}$ where $l \in$ $\mathbb{Z}_{\left[1, q_{i}\right]}, l^{\prime} \in \mathbb{Z}_{\left[1, q_{j}\right]}$ and $(i, j) \in \mathcal{I}_{N}^{2}$, one can write:

$$
\begin{gathered}
A w_{i l}+B\left[F_{j} w_{j l^{\prime}}+g_{j}\right]+\sum_{l=0}^{K} \alpha_{l} \Delta_{l}\left[\left(F_{i} w_{i l}+g_{i}\right)\right. \\
\left.-\left(F_{j} w_{j l^{\prime}}+g_{j}\right)\right] \in \mathcal{X}, \\
\forall(i, j) \in \mathcal{I}_{N}^{2} \text { s.t } x_{k} \in \mathcal{X}_{i} \text { and } x_{k-1} \in \mathcal{X}_{j} .
\end{gathered}
$$

The inclusion (106) is equivalent to the existence of a vector $y_{i j}^{z} \in \mathcal{X}$, that can be expressed as a convex combination of the vertices of $\mathcal{X}$ :

$$
\begin{gathered}
y_{i j}^{z}=V \gamma_{i j}^{z} \text { such that } \gamma_{i j}^{z} \in S_{q}, \\
A w_{i l}+B\left[F_{j} w_{j l^{\prime}}+g_{j}\right]+\sum_{l=0}^{K} \alpha_{l} \Delta_{l}\left[\left(F_{i} w_{i l}+g_{i}\right)\right. \\
\left.-\left(F_{j} w_{j l^{\prime}}+g_{j}\right)\right]=V \gamma_{i j}^{z}, \\
\forall(i, j) \in \mathcal{I}_{N}^{2} \text { s.t } x_{k} \in \mathcal{X}_{i} \text { and } x_{k-1} \in \mathcal{X}_{j} .
\end{gathered}
$$

Let $W_{i} \in \mathbb{R}^{n \times p}$ be the matrix obtained by storing repeatedly as columns the $i^{t h}$ vertex in $\mathcal{W}$. For each vertex in $\mathcal{W}$, the equation:

$$
\begin{gathered}
A W_{i}+B U+\sum_{l=0}^{K} \alpha_{l} \Delta_{l}\left[u^{p w a}\left(W_{i}\right)-U\right]=V L_{i}^{\prime} \\
\forall i \in \mathbb{Z}_{[1, \cdots, p]}, \quad \mathbb{1}^{T} L_{i}^{\prime}=\mathbb{1}^{T}, \quad L_{i}^{\prime} \in \mathbb{R}_{+}^{q \times p} .
\end{gathered}
$$

holds. Finally, (109) leads to the matrix formulations:

$$
\begin{array}{r}
A w_{i l}+B U+\sum_{l=0}^{k} \alpha_{l}\left[\left(F_{i} w_{i l}+g_{i}\right)-U\right]=V \gamma_{i l} \\
\forall(i, j) \in \mathcal{I}_{N}^{2} \text { s.t } x_{k} \in \mathcal{X}_{i} \text { and } x_{k-1} \in \mathcal{X}_{j} .
\end{array}
$$

or, equivalently:

$$
\begin{gathered}
A\left[\begin{array}{llll}
W_{1} & W_{2} & \cdots & W_{p}
\end{array}\right]+B\left[\begin{array}{llll}
U & U & \cdots & U
\end{array}\right]+ \\
\sum_{l=0}^{K} \alpha_{l} \Delta_{l}\left[\begin{array}{llll}
U_{1}-U & U_{2}-U & \cdots & U_{p}-U
\end{array}\right]=V L, \\
\mathbb{1}^{T} L=\mathbb{1}^{T}, \quad L \in \mathbb{R}_{+}^{q \times p^{2}} .
\end{gathered}
$$

Equivalently:

$$
\begin{aligned}
& \underbrace{\left[\begin{array}{lll}
A W O_{p}^{1}+B U & A W O_{p}^{2}+B U & \cdots A W O_{p}^{p}+B U
\end{array}\right]}_{E^{\prime}}+ \\
& \sum_{l=0}^{l=K} \alpha_{l} \Delta_{l} \underbrace{[U O_{p}^{1}-U \underbrace{p}_{p}-U]}_{H^{\prime}}=V L, \\
& E^{\prime}+\sum_{l=0}^{l=k} \alpha_{l} \Delta_{l} H^{\prime}=V L .
\end{aligned}
$$

Finally, the delay margin is obtained as (72) in terms of $\alpha$. Once again, the $d_{m}$ is completely defined due to the isomorphic relation with $d_{m}^{\alpha}$.

\section{APPENDIX D}

PROOF OF PROPOSITION 4.5

We have the following:

- $\epsilon_{k} \in d_{m} \rightarrow \epsilon_{k} \in \mathcal{D}_{m}$

This can be proved by observing that the $\mathcal{D}$-invariance of $\mathcal{X}$ with respect to (16), in the presence of delay $\epsilon_{k} \in d_{m}$, implies that if $x_{i} \in \mathcal{X}, i \in \mathbb{Z}_{[-1,0]}$, then $x_{1} \in \mathcal{X}$. Such an initial condition is equivalent to an extended vector $\xi_{1}=$ $\left[x_{0}^{T} x_{-1}^{T}\right]^{T} \in \mathcal{X} \times \mathcal{X}$, and implies the positive invariance of the set $\mathcal{X} \times \mathcal{X}$ with respect to (18). Consequently, $\epsilon_{k} \in \mathcal{D}_{m}$.

- $\epsilon_{k} \in \mathcal{D}_{m} \rightarrow \epsilon_{k} \in d_{m}$

Starting now from the invariance of $\mathcal{X} \times \mathcal{X}$ with respect to (18), and in order to prove $\mathcal{D}$-invariance of $\mathcal{X}$, one can write:

$$
\begin{aligned}
& x_{k+1}=\left[\begin{array}{ll}
A+\Delta\left(\epsilon_{k}\right) F_{i} & \left(B-\Delta\left(\epsilon_{k}\right)\right) F_{j}
\end{array}\right] \xi_{k}+ \\
& {\left[B g_{j}+\Delta\left(\epsilon_{k}\right)\left(g_{i}-g_{j}\right)\right] \text {; }} \\
& \forall(i, j) \in \mathcal{I}_{N}^{2} \text { such that } \xi_{k} \in \mathcal{X}_{i} \times \mathcal{X}_{j} \text {. }
\end{aligned}
$$

Exploiting the invariance property:

$$
\xi_{k} \in \mathcal{X} \times \mathcal{X} \rightarrow x_{k+1} \in \mathcal{X},
$$

or, equivalently:

$$
\begin{gathered}
{\left[A+\Delta\left(\epsilon_{k}\right) F_{i} \quad\left(B-\Delta\left(\epsilon_{k}\right)\right) F_{j}\right] \xi_{k}+\left[B g_{j}+\Delta\left(\epsilon_{k}\right)\left(g_{i}-g_{j}\right)\right]} \\
\in \mathcal{X}, \forall(i, j) \in \mathcal{I}_{N}^{2} \text { such that } \xi_{k} \in \mathcal{X}_{i} \times \mathcal{X}_{j}
\end{gathered}
$$

as a set inclusion, one can write:

$$
\begin{gathered}
{\left[A+\Delta\left(\epsilon_{k}\right) F_{i} \quad\left(B-\Delta\left(\epsilon_{k}\right)\right) F_{j}\right]\left(\mathcal{X}_{i} \times \mathcal{X}_{j}\right) \oplus} \\
{\left[B g_{j}+\Delta\left(\epsilon_{k}\right)\left(g_{i}-g_{j}\right)\right] \subset \mathcal{X}, \forall(i, j) \in \mathcal{I}_{N}^{2} .}
\end{gathered}
$$

The condition (116) is equivalent to the Minkowski sum:

$$
\begin{gathered}
\left(A+\Delta\left(\epsilon_{k}\right) F_{i}\right) \mathcal{X}_{i} \oplus\left(B-\Delta\left(\epsilon_{k}\right)\right) F_{j} \mathcal{X}_{j} \oplus \\
\left(B g_{j}+\Delta\left(\epsilon_{k}\right)\left(g_{i}-g_{j}\right)\right) \subset \mathcal{X}, \forall(i, j) \in \mathcal{I}_{N}^{2} .
\end{gathered}
$$

thus leading to $\mathcal{D}$-invariance condition for the set $\mathcal{X}$, which subsequently implies that $\epsilon_{k} \in d_{m}$. 


\section{APPENDIX E}

PRoOf of THEOREM 4.6

The $\mathcal{D}$-invariance of the set $\mathcal{X}$ with respect to the original time-varying dynamical system (31) is represented by a set wise relation $\forall \epsilon_{k} \in d_{\text {mult }}$ and $\forall d_{1}>d_{2} \in\left\{d_{d_{\text {min }}, \cdots, d_{\text {max }}}\right\}$ :

$$
\begin{gathered}
A x_{k}+\left(B-\Delta\left(\epsilon_{k}\right)\right) F_{j} x_{k-d_{1}}+\Delta\left(\epsilon_{k}\right) F_{i} x_{k-d_{2}}+ \\
\left(B-\Delta\left(\epsilon_{k}\right)\right) g_{j}+\Delta\left(\epsilon_{k}\right) g_{i} \in \mathcal{X},
\end{gathered}
$$

$\forall(m, i, j) \in \mathcal{I}_{N}^{3}$ s.t $x_{k} \in \mathcal{X}_{m}$ and $x_{k-d_{2}} \in \mathcal{X}_{i}$ and $x_{k-d_{1}} \in \mathcal{X}_{j}$, which, by exploiting the convex combinations of extreme realizations of $\Delta\left(\epsilon_{k}\right)$, is equivalent to:

$$
\begin{gathered}
A x_{k}+B\left(F_{j} x_{k-d_{1}}+g_{j}\right)+\sum_{l=0}^{K} \alpha_{l} \Delta_{l}\left[\left(F_{i} x_{k-d_{2}}+g_{i}\right)\right. \\
\left.-\left(F_{j} x_{k-d_{1}}+g_{j}\right)\right] \in \mathcal{X},
\end{gathered}
$$

$\forall(m, i, j) \in \mathcal{I}_{N}^{3}$ s.t $x_{k} \in \mathcal{X}_{m}$ and $x_{k-d_{2}} \in \mathcal{X}_{i}$ and $x_{k-d_{1}} \in \mathcal{X}$ with $\alpha_{l}, l \in \mathbb{Z}_{[0, K]}$ being the elements of a vector $\alpha \in S_{K+1}$. By expressing the state vectors $x_{k} \in \mathcal{X}_{m}$ and $x_{k-d_{1}} \in \mathcal{X}_{j}$ and $x_{k-d_{2}} \in \mathcal{X}_{i}$

$$
\begin{gathered}
x_{k}=\sum_{l=1}^{q_{m}} \lambda_{l} w_{m l} \text { for } \lambda \in S_{q_{m}}, \\
x_{k-d_{2}}=\sum_{l^{\prime}=1}^{q_{i}} \lambda_{l^{\prime}}^{\prime} w_{i l^{\prime}} \text { for } \lambda^{\prime} \in S_{q_{i}}, \\
x_{k-d_{1}}=\sum_{l^{\prime \prime}=1}^{q_{j}} \lambda_{l^{\prime \prime}}^{\prime \prime} w_{j l^{\prime \prime}} \text { for } \lambda^{\prime \prime} \in S_{q_{j}} .
\end{gathered}
$$

Then for a given set of vertices in $w_{m l}, w_{i l^{\prime}}, w_{j l^{\prime \prime}}$ where $l \in$ $\mathbb{Z}_{\left[1, q_{m}\right]}, l^{\prime} \in \mathbb{Z}_{\left[1, q_{i}\right]}, l^{\prime \prime} \in \mathbb{Z}_{\left[1, q_{j}\right]}$ and $(m, i, j) \in \mathcal{I}_{N}^{3}$, one can write:

$$
\begin{gathered}
A w_{m l}+B\left[F_{j} w_{j l^{\prime \prime}}+g_{j}\right]+\sum_{l=0}^{K} \alpha_{l} \Delta_{l}\left[\left(F_{i} w_{i l^{\prime}}+g_{i}\right)\right. \\
\left.-\left(F_{j} w_{j l^{\prime \prime}}+g_{j}\right)\right] \in \mathcal{X},
\end{gathered}
$$

$\forall(m, i, j) \in \mathcal{I}_{N}^{3}$ s.t $x_{k} \in \mathcal{X}_{m}$ and $x_{k-d_{2}} \in \mathcal{X}_{i}$ and $x_{k-d_{1}} \in \mathcal{X}_{j}$.

The inclusion (118) is equivalent to the existence of a vector $y_{m i j}^{z} \in \mathcal{X}$ which can be expressed as a convex combination of the vertices of $\mathcal{X}$ :

$$
\begin{gathered}
y_{m i j}^{z}=V \gamma_{m i j}^{z} \text { such that } \gamma_{m i j}^{z} \in S_{q}, \\
A w_{m l}+B\left[F_{j} w_{j l^{\prime \prime}}+g_{j}\right]+\sum_{l=0}^{K} \alpha_{l} \Delta_{l}\left[\left(F_{i} w_{i l^{\prime}}+g_{i}\right)\right. \\
\left.-\left(F_{j} w_{j l^{\prime \prime}}+g_{j}\right)\right]=V \gamma_{m i j}^{z},
\end{gathered}
$$

$\forall(m, i, j) \in \mathcal{I}_{N}^{3}$ s.t $x_{k} \in \mathcal{X}_{m}$ and $x_{k-d_{2}} \in \mathcal{X}_{i}$ and $x_{k-d_{1}} \in \mathcal{X}_{j}$

Let $W_{m}, W_{i} \in \mathbb{R}^{n \times p}$ be the matrices obtained by storing repeatedly as columns the $m^{t h}$ and $i^{t h}$ vertices, respectively, in $\mathcal{W}$. For each pair of vertices in $\mathcal{W}$, the equation:

$$
\begin{aligned}
& A W_{m}+B U+\sum_{l=0}^{K} \alpha_{l} \Delta_{l}\left[u^{p w a}\left(W_{i}\right)-U\right]=V L_{m i}^{\prime}, \\
& \forall(m, i) \in \mathbb{Z}_{[1, \cdots, p]}^{2}, \quad \mathbb{1}^{T} L_{m i}^{\prime}=\mathbb{1}^{T}, \quad L_{m i}^{\prime} \in \mathbb{R}_{+}^{q \times p}
\end{aligned}
$$

holds. Furthermore, for each vertex with the index $m$ in $\mathcal{W}$, the following holds:

$$
\begin{gathered}
A\left[W_{m} \cdots W_{m}\right]+B[U \cdots U]+ \\
\sum_{l=0}^{K} \alpha_{l} \Delta_{l}\left[U_{1}-U \cdots U_{p}-U\right]=V L_{m}^{\prime \prime}, \\
\forall m \in \mathbb{Z}_{[1, \cdots, p]}, \quad \mathbb{1}^{T} L_{m}^{\prime \prime}=\mathbb{1}^{T}, \quad L_{m}^{\prime \prime} \in \mathbb{R}_{+}^{q \times p^{2}} .
\end{gathered}
$$

Finally, the condition (122) leads to the matrix formulation:

$$
\begin{gathered}
A[\underbrace{W_{1} \cdots W_{1}}_{{ }^{\prime}{ }^{\prime} \text { times }} \cdots \cdots \underbrace{W_{p} \cdots W_{p}}_{{ }^{\prime} p^{\prime} \text { times }}]+ \\
B[\underbrace{U \cdots U}_{{ }^{\prime} p^{\prime} \text { times }} \cdots \cdots \underbrace{U \cdots U}_{{ }^{\prime} p^{\prime} \text { times }}]+ \\
\sum_{l=0}^{K} \alpha_{l} \Delta_{l}[\underbrace{U_{1}-U \cdots U_{p}-U}_{{ }^{\prime} p^{\prime} \text { times }} \cdots \underbrace{U_{1}-U \cdots U_{p}-U}_{{ }^{\prime} p^{\prime} \text { times }}]= \\
\mathbb{1}^{T} L=\mathbb{1}^{T}, \quad L \in \mathbb{R}_{+}^{q \times p^{3}},
\end{gathered}
$$

where $W_{i}=W O_{p}^{i}$ and $U_{i}=U O_{p}^{i}$.

Equivalently:

$$
E^{\prime \prime}+\sum_{l=0}^{l=k} \alpha_{l} \Delta_{l} H^{\prime \prime}=V L
$$

Finally, the intersample delay margin for fixed pair $d_{1}, d_{2} \in$ $\left\{d_{\min }, \cdots, d_{\max }\right\}$ is obtained in terms of $\alpha$ as in (72). $d_{\text {mult }}$ is obtained afterwards exploiting the third claim of Proposition 4.7 and Corollary 4.8.

\section{ACKNOWLEDGMENT}

The research leading to these results has benefited from the financial support of the European Union's $7^{\text {th }}$ Framework Programme under EC-GA No. 607957 TEMPO - Training in Embedded Predictive Control and Optimization.

\section{REFERENCES}

[1] W. Heemels, N. Van de Wouw, R. Gielen, M. Donkers, L. Hetel, S. Olaru, M. Lazar, J. Daafouz, and S. Niculescu, "Comparison of overapproximation methods for stability analysis of networked control systems," in Proceedings of the $13^{\text {th }}$ ACM International Conference on Hybrid systems: computation and control, 2010, pp. 181-190.

[2] W. Michiels and S.-I. Niculescu, Stability and Stabilization of TimeDelay Systems (Advances in Design \& Control). Philadelphia, PA, USA: Society for Industrial and Applied Mathematics, 2007.

[3] K. Gu, V. L. Kharitonov, and J. Chen, Stability of time-delay systems. Birkhäuser, Boston, USA: Springer Science + Business Media, LLC, 2003.

[4] J. Maciejowski, Predictive Control with Constraints. England.: Prentice Hall, 2002.

[5] T. A. Johansen and A. Grancharova, Explicit Nonlinear Model Predictive Control. Springer-Verlag Berlin Heidelberg, 2012.

[6] A. Bemporad, M. Morari, V. Dua, and E. N. Pistikopoulos, "The explicit linear quadratic regulator for constrained systems," Automatica, vol. 38, no. 1, pp. 3-20, 2002.

[7] T. A. Johansen and A. Grancharova, "Approximate explicit constrained linear model predictive control via orthogonal search tree," IEEE Transactions on Automatic Control, vol. 48, no. 5, pp. 810-815, 2003.

[8] M. Johansson and A. Rantzer, "Computation of piecewise quadratic lyapunov functions for hybrid systems," IEEE Transactions on Automatic Control, vol. 43, no. 4, pp. 555-559, 1998. 
[9] M. Hovd and S. Olaru, "Relaxing PWQ lyapunov stability criteria for PWA systems," Automatica, vol. 49, no. 2, pp. 667-670, 2013.

[10] R. Iervolino, F. Vasca, and L. Iannelli, "Cone-copositive piecewise quadratic lyapunov functions for conewise linear systems," IEEE Transactions on Automatic Control, vol. 60, no. 11, pp. 3077-3082, 2015.

[11] F. Blanchini and S. Miani, Set-theoretic methods in control. Springer, 2008.

[12] G. Bitsoris, "Positively invariant polyhedral sets of discrete-time linear systems," International Journal of Control, vol. 47, no. 6, pp. 17131726, 1988.

[13] W. Lombardi, "Constrained control for time-delay systems." Ph.D. dissertation, Supélec, 2011.

[14] M. B. G. Cloosterman, N. van de Wouw, W. M. P. H. Heemels, and H. Nijmeijer, "Robust stability of networked control systems with timevarying network-induced delays," in $45^{\text {th }}$ IEEE Conference on Decision and Control, 2006, pp. 4980-4985.

[15] Z. Wang, J. Sun, and J. Chen, "A new polytopic approximation method for networked systems with time-varying delay," IEEE Transactions on Circuits and Systems, vol. 63, no. 9, pp. 843-847, 2016.

[16] R. Gielen, S. Olaru, M. Lazar, W. Heemels, N. Van de Wouw, and S. Niculescu, "On polytopic inclusions as a modeling framework for systems with time-varying delays," Automatica, vol. 46, no. 3, pp. 615619, 2010.

[17] S. Olaru, N. Stanković, G. Bitsoris, and S.-I. Niculescu, "Low complexity invariant sets for time-delay systems: A set factorization approach," in Low-Complexity Controllers for Time-Delay Systems. Springer, 2014, pp. $127-139$.

[18] N. A. Nguyen, S. Olaru, P. Rodriguez-Ayerbe, G. Bitsoris, and M. Hovd "Explicit robustness and fragility margins for linear discrete systems with piecewise affine control law," Automatica, vol. 68, pp. 334-343, 2016.

[19] M.-T. Laraba, S. Olaru, and S.-I. Niculescu, "Analysis of pwa control of discrete-time linear dynamics in the presence of variable time-delay," in 55th IEEE Conference on Decision and Control, 2016, pp. 567-572.

[20] F. Blanchini, "Set invariance in control," Automatica, vol. 35, pp. $1747-$ 1767, 1999.

[21] W. Lombardi, S. Olaru, S.-I. Niculescu, and L. Hetel, "A predictive control scheme for systems with variable time-delay," International Journal of Control, vol. 85, no. 7, pp. 915-932, 2012.

[22] A. Bemporad, M. Morari, V. Dua, and E. Pistikopoulos, "The explicit linear quadratic regulator for constrained systems," Automatica, vol. 38 , pp. 3-20, 2002.

[23] S. Olaru and S.-I. Niculescu, "Predictive control for systems with delay and constraints," in Proceedings of IFAC World Congress, 2008.

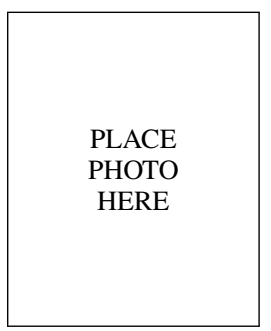

Mohammed-Tahar Laraba received his M.Sc. degree in Control and Signals Processing from ParisSud University, France in 2014. Thereafter, he started working towards a Ph.D. degree in the Laboratory of Signals and Systems at CentraleSupélec. $\mathrm{He}$ is working as an Early Stage Researcher (ESR) in Marie Curie Initial Training Network on Embedded Model Predictive Control and Optimization (ITNTEMPO). His research interests include analysis and control synthesis for dynamical systems under constraints, set-valued systems analysis and synthesis, time-delay systems, model predictive control.

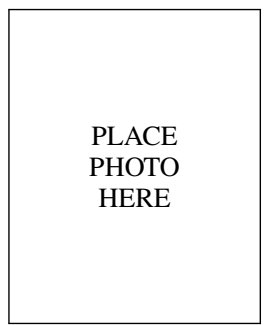

Silviu-Iulian Niculescu received the B.Sc. degree from the Polytechnical Institute of Bucharest, Romania, the M.Sc., Ph.D. degrees, in automatic control, from INP Grenoble, France and the French habilitation from Universit Technologique de Compigne, France. From September 2006, he joined L2S (Laboratory of Signals and Systems), Gif-sur-Yvette, where he is currently a CNRS research director (senior researcher) and the head of the laboratory. Dr. Niculescu is the author or co-author of 9 books and more than 400 book chapters or scientific papers. Since 2012, he is the founding editor and the scientific responsible of a new Springer (book) series: Advances in Delays in Dynamics (ADD@S). Dr. Niculescu was awarded the CNRS Silver and Bronze Medals for scientific research, and the Ph.D. Thesis Award from INP Grenoble (France) in 2011, 2001, and 1996, respectively. His research interests include delay systems, robust control, operator theory, and numerical methods in optimization, and their applications to the design of engineering systems.

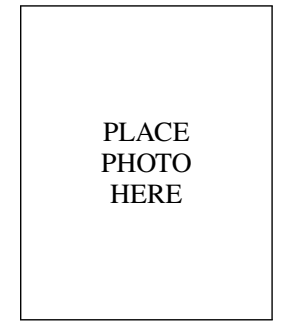

Sorin Olaru is a Professor in CentraleSupélec, member of the CNRS Laboratory of Signals and Systems and of the INRIA team DISCO, all these institutions being part of the Paris-Saclay University in France. His research interests are encompassing the optimization-based control design, set-theoretic characterization of constrained dynamical systems as well as the numerical methods in control. $\mathrm{He}$ is currently involved in research projects related to embedded predictive control, fault tolerant control and time-delay systems. 